\title{
Chromatin tethering effects of hNopp140 are involved in the spatial organization of nucleolus and the rRNA gene transcription
}

\author{
Yi-Tzang Tsai $\cdot$ Chen-I Lin $\cdot$ Hung-Kai Chen $\cdot$ Kuo-Ming Lee $\cdot$ \\ Chia-Yi Hsu · Shun-Jen Yang · Ning-Hsing Yeh
}

Received: 5 October 2007 / Accepted: 23 November 2007/Published online: 6 February 2008

(C) National Science Council Taipei 2008

\begin{abstract}
The short arms of five human acrocentric chromosomes contain ribosomal gene (rDNA) clusters where numerous mini-nucleoli arise at the exit of mitosis. These small nucleoli tend to coalesce into one or a few large nucleoli during interphase by unknown mechanisms. Here, we demonstrate that the $\mathrm{N}$ - and C-terminal domains of a nucleolar protein, hNopp140, bound respectively to $\alpha$-satellite arrays and rDNA clusters of acrocentric chromosomes for nucleolar formation. The central acidicand-basic repeated domain of hNopp140, possessing a weak self-self interacting ability, was indispensable for hNopp140 to build up a nucleolar round-shaped structure. The N- or the C-terminally truncated hNopp140 caused nucleolar segregation and was able to alter locations of the rDNA transcription, as mediated by detaching the rDNA repeats from the acrocentric $\alpha$-satellite arrays. Interestingly, an hNopp140 mutant, made by joining the N- and C-terminal domains but excluding the entire central repeated region, induced nucleolar disruption and global chromatin condensation. Furthermore, RNAi knockdown of hNopp140 resulted in dispersion of the rDNA and acrocentric $\alpha$-satellite sequences away from nucleolus that was accompanied by rDNA transcriptional silence. Our findings indicate that hNopp140, a scaffold protein, is involved in the nucleolar assembly, fusion, and maintenance.
\end{abstract}

Keywords Acrocentric chromosomes - Alpha-satellite DNA · Centromere · Nopp140 · Nucleolus · rDNA

Y.-T. Tsai · C.-I. Lin · H.-K. Chen · K.-M. Lee · C.-Y. Hsu • S.-J. Yang · N.-H. Yeh $(\bowtie)$

School of Life Science, Institute of Microbiology and Immunology, National Yang-Ming University, 155 Li-Nong

Street Sec. 2, Taipei 112, Taiwan

e-mail: yphcsl@ym.edu.tw

\section{Introduction}

The human diploid genome contains $\sim 400$ ribosomal RNA gene (rDNA) copies, each of which is composed of a $13 \mathrm{~kb}$ transcribed region and a $30 \mathrm{~kb}$ intergenic spacer. These units are arranged in a mosaic of canonical and palindromic repeats [1], collectively spanning megabases on each of the short arms of the five acrocentric chromosomes 13, 14, 15, 21 and 22, at positions termed nucleolar organizer regions (NORs) [2, 3]. The remaining parts of the acrocentric short arms are composed of interspersed arrays of different repetitive satellite DNA, which extend to the centromeric and telomeric regions [4-7]. As cells exit from mitosis, transcription of the rDNA loci leads to the formation of numerous mini-nucleoli around the active NORs. These small nucleoli tend to coalesce into a single or a few large nucleoli in the interphase nucleus by mechanisms that are currently unknown [8-12]. Evidence has shown that the rDNA and the centromeric sequences of acrocentric chromosomes are closely juxtaposed in the interphase nucleus [13-15], and that several NOR-bearing chromosomes are involved in the development of one nucleolus [16]. Notably, at any given time only a subset of the rDNA is actively transcribed [17], and most of the transcriptionally silent NORs are also included in nucleoli [18]. Thus, in addition to the rDNA transcription and ribosome biogenesis, in which both activities are believed to be essential for maintaining the nucleolar integrity [19], certain unknown structural elements must exist for tethering the short arms of the five acrocentric chromosomes into a nearby spatial location in interphase to facilitate the formation of a large nucleolar territory.

The nucleolar territory in higher eukaryotic cells consists of three morphologically distinct zones as observed by electron microscopy. Pale fibrillar centers (FCs) are surrounded by dense fibrillar components (DFCs) to form 
many round-shaped islets, which in turn are embedded in granular components (GCs) [20]. The rDNA repeats localize to the FCs and/or DFCs that represent interphase NORs and thereby sites of transcription. In the DFCs, nascent pre-rRNA transcripts give rise to the "Christmas trees" structure [21, 22] and subject to the early processing events [23, 24]. Subsequently, these transcripts move to the GCs for the late processing steps followed by the assembly of the mature rRNA and ribosomal proteins into preribosomal particles [25]. On the light microscopy level, the RNA polymerase I (pol I) or the rDNA foci combined with the nucleolar Br-UTP incorporation sites, equivalent to the FCs and DFCs, can be visualized as intense dots embedded within the nucleolar body [21, 26].

The Nopp140 homologous proteins of rat, human, frog and fruit fly distribute in the nucleolus in a dotted pattern; their sequences show a three-domain feature, a large central region with alternating acidic and basic motifs surrounded by the small N- and C-terminal segments [27-30]. The C-terminal domain is conserved in all Nopp140 homologs across a broad range of eukaryotes [31]. In contrast, the $\mathrm{N}$-terminal domain is highly conserved in mammals but divergent in other species, and even absent in the budding yeast [32]. Reports based on coimmunoprecipitation indicate that Nopp140 associates with both FC and DFC components, representing by pol I and small nucleolar ribonucleoprotein (snoRNP) complexes [33-35]. It has been suggested that Nopp140 is not essential for the box H/ACA snoRNP-mediated pseudouridylation of rRNA [35] and that the involvement of Nopp140 in U3 snoRNP biogenesis occurs in nucleoplasm but not in nucleolus [36]. Nopp140 is known to be present predominantly in the nucleolus and its precise roles in the early ribosome biogenesis remain unclear. Nevertheless, there are clues that functions of Nopp140 may relate to the nucleolar structural organization. For example, deletion mutants of Nopp140 with dominantnegative effects can alter the normal distribution of nucleolar components and arrest the rDNA transcription [33, 34, 37]. Here, we report that human Nopp140 (hNopp140) relies respectively on its $\mathrm{N}$ - and $\mathrm{C}$-terminal domains for tethering the $\alpha$-satellite DNA and the rDNA repeats of acrocentric chromosomes to facilitate the nucleolar formation and rDNA transcription. These findings imply that Nopp140, as a nucleolar architectural scaffold, is involved in the assembly of FCs and DFCs.

\section{Materials and methods}

Antibodies

Monoclonal antibodies (mAbs) used included M2 (IgG1; anti-Flag; Sigma), 9E10 (IgG1; anti-c-Myc; Santa Cruz), CP2 (IgG2a; anti-hNopp140), HC2 (IgG2b;
anti-hNopp140), JY1 (IgG1; anti-hNopp140), F9 (IgG1; anti-upstream binding factor; Santa Cruz), Tau-1 (IgG2a; anti-nuclear tau; a gift from Lester I. Binder, Northwestern University Medical School) [38], and BU33 (IgG1; anti-BrdUTP; Sigma). For generating mAb to RPA194 (the largest subunit of pol I; GenBank database, U33460), a pair of primers 5'-GCTTTCAGGAACGTAAACACTCGAAG-3' and 5'-TGCTCTGTACTGTCACTTGGAACTGC-3' were used to amplify a $1.2 \mathrm{~kb}$ fragment of human RPA194 cDNA from human K562 cell line cDNA library (Clontech). The polymerase chain reaction (PCR) product was cloned into an expression vector pGEX-3X (Amrad). A $72 \mathrm{kDa}$ glutathione S-transferase fusion protein of RPA194 (amino acids 13641772) was expressed and purified by glutathione-agarose beads (Amrad). The purified fusion protein was used as antigen to generate hybridomas as described [28]. Monoclonal antibody JY10 (IgG2a; anti-RPA194) was obtained.

\section{Mammalian expression constructs and transfection}

Expression vectors pFLAG-CMV2 (Sigma) and pCMVTag 3 (Stratagene) were used to express protein fused $\mathrm{N}$ terminally with a tag, Flag or c-Myc, respectively. The Flag-tagged full-length and deletion mutants DM1, and DM5 of hNopp140 have been constructed previously [33]. Flag-tagged DM2, DM3, DM4, and DM8 were created respectively by inserting the StuI-EcoRI $(2.20 \mathrm{~kb})$, StuIHindIII (1.57 kb), HindIII-HindIII $(0.57 \mathrm{~kb})$, and BanIIEcoRI $(0.83 \mathrm{~kb})$ fragments from hNopp140 cDNA into the pFLAG-CMV2 vector in frame with the Flag at the $5^{\prime}$ end. DM7 was generated by restriction enzyme digestion of the full-length hNopp140 cloned in pFLAG-CMV2 to remove the hNopp140 cDNA sequence downstream of the StuI. For construction of the DM9, DM10, DM11, PCR amplified fragments encoding various lengths of the C-terminal domain of hNopp140 (see Fig. 2a) were ligated in frame to pFLAG-CMV2 containing the coding region of hNopp140 from the beginning downstream to the StuI site. DM6 was constructed by PCR methods with appropriate pairs of primers. The inserts containing the full-length hNopp140 cDNA and DM3 from the pFLAG-CMV2 vector were then subcloned in frame to the pCMV-Tag 3. All constructs have been sequence-confirmed.

For transfection studies, Lipofectamine (GibcoBRL) method was applied to HeLa cells as described previously [33], and calcium phosphate precipitation method was used for 293T cells similar to that reported before [39].

In situ hybridization and detection

The DNA fragment encompassing the rDNA promoter region from -321 to -22 was amplified from the whole 
genomic DNA of HeLa cells by PCR using a pair of primers: $5^{\prime}$-CTCGTGTGTCCCGGTCGTAG- $3^{\prime}$ and $5^{\prime}$-TG CCGACTCGGAGCGAAAG-3' (GenBank database, U13369), and it was inserted into the pGEM $^{\circledR}$-T Easy vector (Promega). Similarly, the $287 \mathrm{bp}$ acrocentric $\alpha$-satellite DNA was cloned by using a pair of primers: $5^{\prime}$-A TTTGGAGGCCTTTGTGGCTATGG- $3^{\prime}$ and $5^{\prime}$-GGCC TCAAAACGCTCCAAGTATCC-3' [7] (GenBank database, X55368), and the 332 bp chromosome 9-specific $\alpha$-satellite DNA by a pair of primers: $5^{\prime}$-CCTATGATGAAAAAGGTAATATCTTCCC- $3^{\prime}$ and $5^{\prime}$-CCTGAAA GCGCTTAAAACGTCCGCTT-3' [40] (GenBank database, M64320). To label the rDNA and the $\alpha$-satellite probes with biotin, PCR was performed in the presence of biotin-16-dUTP (Roche) using the plasmids above as templates and the same pairs of primer sets. The probes were then eluted and resuspended in hybridization buffer ( $2 \times$ SSC, standard saline citrate [pH 7.2]; 50\% formamide; $10 \%$ dextran sulfate). Prior to in situ hybridization, the DNA probes were denatured at $90^{\circ} \mathrm{C}$ for $5 \mathrm{~min}$, and placed on ice immediately.

Hybridization was performed with modifications of the procedure described previously $[41,42]$. HeLa cells grown on coverslips were fixed with $3.7 \%$ formaldehyde in PBS for $20 \mathrm{~min}$ at room temperature, permeabilized with $-20^{\circ} \mathrm{C}$ acetone for $3 \mathrm{~min}$, and washed once in PBS. The fixed cells were then denatured in alkalified PBS ( $\mathrm{pH} 12.5)$ for $2 \mathrm{~min}$ and immediately dehydrated through $70 \%, 80 \%, 90 \%$, and $100 \%$ of ice-cold ethanol, 1 min for each, and dried in air. The hybridization mixture containing $200 \mathrm{ng}$ of the biotinylated rDNA or $\alpha$-satellite probe in $8 \mu$ of hybridization buffer was applied to a slide, which was then covered by the treated coverslip with cells, and sealed with rubber cement. The probe-and-cell loaded slides were heated for further denaturation on the $80^{\circ} \mathrm{C}$ hot plate for $5 \mathrm{~min}$. After a brief cool-down, samples were subjected to the microwave activation utilizing a conventional oven with $160 \mathrm{~W}$ of power for $5 \mathrm{~min}$. One liter of water was also placed in the microwave to prevent the ebullition of the hybridization liquid. After hybridization, the coverslips were washed in $0.2 \times \mathrm{SSC}$ at $43^{\circ} \mathrm{C}$ for three times, each for $1 \mathrm{~min}$ for the rDNA probe or $2 \mathrm{~min}$ for the acrocentric $\alpha$-satellite probe; or in $0.1 \times \mathrm{SSC}$ at $50^{\circ} \mathrm{C}$ for three times, each for $1 \mathrm{~min}$ for the chromosome 9 -specific $\alpha$-satellite probe. The cells were then subjected to the combined immunofluorescence staining by using primary antibodies, anti-Flag, antihNopp140, anti-pol I, or anti-UBF, and then the secondary antibody, fluorescein isothiocyanate (FITC)-conjugated goat anti-mouse $\operatorname{IgG}$ (Caltag). UltraAvidin ${ }^{\mathrm{TM}}$-Rhodamine (Leinco) for detecting the hybridized DNA probe was applied together with the secondary antibody. Hoechst $33258(0.4 \mu \mathrm{g} / \mathrm{ml}$; Sigma) was also added at the last step of staining.
Double immunofluorescence microscopy

The procedure for indirect immunofluorescence staining was according to that described previously [33]. Different sets of antibodies were used for double immunofluorescence. For costaining of pol I with hNopp140 (or UBF), anti-pol I, JY10, was detected by FITC-conjugated goat anti-mouse IgG2a (Caltag), while anti-hNopp140, JY1, (or anti-UBF, F9), was probed by biotin-conjugated goat antimouse IgG1 (Caltag) followed by UltraAvidin ${ }^{\mathrm{TM}}$-Rhodamine. For costaining of ectopically expressed Flag-tagged protein with the endogenous hNopp140, the primary antibody, anti-Flag, was detected either by FITC-conjugated goat anti-mouse IgG1 (Caltag), or by biotin-conjugated goat anti-mouse IgG1 followed by UltraAvidin ${ }^{\mathrm{TM}}$-Rhodamine; while for matching the green-and-red colors, the primary anti-hNopp140 antibody, CP2, was traced by biotin-conjugated goat anti-mouse $\mathrm{IgG} 2 \mathrm{a}$ (Caltag) followed by UltraAvidin ${ }^{\mathrm{TM}}$-Rhodamine, or by FITC-conjugated goat anti-mouse IgG2a. This protocol was also applied to costain the Flag-tagged protein and the endogenous pol I by anti-Flag (IgG1) and JY10 (IgG2a). Hoechst 33258 $(0.4 \mu \mathrm{g} / \mathrm{ml})$ was added to the last staining solution.

Samples mounted in antifade fluid $(1 \mathrm{mg} / \mathrm{ml} \mathrm{p}$-phenylenediamine, $150 \mathrm{mM}$ Tris-HCl [pH 8.8], 90\% glycerol) were examined under an Olympus BX50 or BX51 epifluorescence microscope equipped with $40 \times / 0.75$ and $100 \times / 1.30$ oil-immersion objectives, and photographed on Kodak Elite Chrome 400 films or to digital images through CoolSNAP $f x$ CCD monochrome camera (Taiwan Instrument Co.), respectively. The images on the films were digitalized with a film scanner (DiMAGE Scan Elite II, Konica Minolta). The software Photoshop CS (Adobe) was used for figure assembly.

\section{Cell lysis, immunoprecipitation, and immunoblotting}

The $293 \mathrm{~T}$ cells were lysed in the lysis buffer $(50 \mathrm{mM}$ Tris$\mathrm{HCl}$ [pH 7.4], $150 \mathrm{mM} \mathrm{NaCl}, 1 \%$ Triton X-100, $2 \mathrm{mM}$ dithiothreitol, $1 \mathrm{mM}$ phenylmethylsulfonyl fluoride, $1 \mu \mathrm{g} /$ $\mathrm{ml}$ leupeptin, $1 \mu \mathrm{g} / \mathrm{ml}$ aprotinin) at $4^{\circ} \mathrm{C}$ for $1 \mathrm{~h}$. After a brief sonication, lysates were clarified by centrifugation $(15,700 \mathrm{~g}$, $4^{\circ} \mathrm{C}$ for $20 \mathrm{~min}$ ) and subjected to immunoblotting analysis. The 293T cell lysates were also for immunoprecipitation using M2 affinity gel (anti-Flag; Sigma) or 9E10 conjugated agarose (anti-c-Myc; Santa Cruz), followed by immunoblotting with anti-hNopp140, CP2, or anti-tag antibodies, as described previously [33]. In some experiments as indicated, $25 \mu \mathrm{g} / \mathrm{ml}$ DNase (Promega) was added to the mixtures of the cell lysate and the M2 affinity beads. Then, samples were tumbling at $4{ }^{\circ} \mathrm{C}$ overnight for antigen-antibody interaction and DNase digestion. 
Chromatin immunoprecipitation (ChIP) assay

ChIP assays were according to methods described previously [43]. Briefly, HeLa cells, or 293T cells with or without transfection, were exposed to formaldehyde at a final concentration of $1 \%$ added directly to the tissue culture medium for $10 \mathrm{~min}$ at room temperature, then, followed by incubation with $125 \mathrm{mM}$ glycine for $5 \mathrm{~min}$ and cold PBS wash twice to stop the cross-linking reaction. Cell pellets were lysed in the lysis buffer as described above $\left(2 \times 10^{5}\right.$ cells $\left./ \mathrm{ml}\right)$. The cell lysates, $1 \mathrm{ml}$ per aliquot, were sonicated using a microtip until the DNA fragments were $0.5-1 \mathrm{~kb}$ in length. The chromatin samples were subjected to centrifugation $\left(15,700 \mathrm{~g}, 4^{\circ} \mathrm{C}\right.$ for $\left.20 \mathrm{~min}\right)$ to remove debris. A small aliquot of the supernatant was taken as the input sample and stored at $-70^{\circ} \mathrm{C}$ until use for PCR. About $1 \mathrm{ml}$ of the supernatant was immunoprecipitated with the protein A-Sepharose beads preadsorbed with anti-hNopp140, HC2, or with anti-Flag M2 affinity gels, according to methods described before [33], except that $10 \mu \mathrm{g} / \mathrm{ml}$ salmon sperm DNA (Invitrogen) was added to the reaction mixtures and that the immunocomplex-coupled beads were washed extensively with NET buffer (0.5 mM EDTA, $50 \mathrm{mM}$ Tris- $\mathrm{HCl}$ [pH 7.4], $150 \mathrm{mM}$ $\mathrm{NaCl}, 0.25 \% \mathrm{NP}-40$ ). To isolate the precipitated DNA, the immunocomplexes were disrupted with $50 \mu$ l elution buffer ( $1 \%$ sodium dodecyl sulfate, $100 \mathrm{mM} \mathrm{NaHCO} 3$ ) twice at $100^{\circ} \mathrm{C}$ for $10 \mathrm{~min}$ each. The pooled eluates were heated at $65^{\circ} \mathrm{C}$ for $8 \mathrm{~h}$ to reverse the formaldehyde crosslink. The decrosslinked samples were extracted with phenol/chloroform/isoamylalcohol (volume ratio 25:24:1) and then ethanol precipitated. DNA pellets derived from $2 \times 10^{5}$ cells were dissolved in $25 \mu \mathrm{l}$ water and used as a template for PCR with the specific primer sets described above for the rDNA promoter, the $\alpha$-satellite of chromosomes 13,14 , and 21 , or the $\alpha$-satellite of chromosome 9 . PCRs were conducted under the following conditions: $95^{\circ} \mathrm{C}$ for $5 \mathrm{~min}$, followed by 25 cycles of $95^{\circ} \mathrm{C}$ for $30 \mathrm{~s}$, and then $55^{\circ} \mathrm{C}$ (rDNA promoter), $57^{\circ} \mathrm{C}(\alpha$-satellite of chromosomes $13,14,21)$, or $58^{\circ} \mathrm{C}(\alpha$-satellite of chromosome 9) for $30 \mathrm{~s}$, followed by $72^{\circ} \mathrm{C}$ for $1 \mathrm{~min}$. The final round of extension for all reactions was $72^{\circ} \mathrm{C}$ for $7 \mathrm{~min}$. PCR products were resolved in $2 \%$ argarose gels, and stained with ethidium bromide. The mock controls were performed with the same procedures except that NS1 myeloma cell culture supernatant was added to the Protein A-Sepharose beads.

Electrophoretic mobility shift assay (EMSA)

The DNA insert encompassing rDNA promoter region from -321 to -22 in the pGEM-T Easy vector was released by EcoRI digestion and gel-purified. To label the rDNA probe with biotin, Klenow fragment (New England BioLabs) was used to fill-in both recessive $3^{\prime}$-ends in the presence of biotin-16-dUTP. The $287 \mathrm{bp}$ acrocentric $\alpha$ satellite DNA was labeled similarly. To prepare the immunopurified proteins, lysis of normal HeLa cells or 293 T cells expressing DM7 or DM8 was performed with method described above. After immunoprecipitation with antibodies against hNopp140, UBF, tau, or Flag tag, the immunocomplexes were dissociated from affinity beads by $0.1 \mathrm{M}$ glycine ( $\mathrm{pH} 3.0$ ), and then immediately neutralized in a $1 / 10$ volume of $1 \mathrm{M}$ Tris- $\mathrm{HCl}(\mathrm{pH} 8.0)$.

For DNA binding reaction, the pull-down protein complexes were mixed with $0.4 \mu \mathrm{g}$ of labeled DNA probe and $2 \mu \mathrm{g}$ of salmon sperm DNA in binding buffer $(17 \mathrm{mM}$ HEPES [pH 7.9], $14.5 \%$ glycerol, $0.42 \mathrm{mM}$ EDTA, $0.3 \mathrm{mM}$ DTT, $10 \mathrm{mM} \mathrm{KCl}, 6.25 \mathrm{mM} \mathrm{MgCl}_{2}$ ) for $30 \mathrm{~min}$ at room temperature. To verify the specific interaction, unlabeled probe competitor was also added into the reaction mix in different concentrations as indicated. The reaction products were resolved on a $4 \%$ native polyacrylamide gel, and then transferred to Immobilon-P PVDF membrane (MILLIPORE) by electroblot. Biotin-labeled moieties were detected with avidin-peroxidase conjugate (Sigma) and chemiluminescent substrate.

siRNA treatment and in situ run-on transcription assay

A siRNA duplex, synthesized by Sigma Proligo, was used to down regulate the expression levels of hNopp140. The duplex was designed as 21-mers with $3^{\prime}$-dTdT overhang, targeting to the position 468-486 (5'-GCCAAAGCUC CUCCUAAGA-3') of hNopp140 mRNA. For each Lipofectamine transfection, siRNA was applied into HeLa cell culture to a final concentration of $10 \mathrm{nM}$. As a negative control, cells were treated with siCONTROL Non-Targeting siRNA (Dharmacon), which was bioinformatically designed with at least 4 mismatches to any known human genes. Cells were then analyzed $72 \mathrm{~h}$ after transfection.

In situ run-on transcription assay was performed as previously described [33], except the Br-UTP incorporation modified as for $10 \mathrm{~min}$.

\section{Results}

The rDNA repeats colocalized with hNopp140

Nucleolus is disintegrated at the onset of mitosis and reformed during mitotic exit [10]; these dynamic changes are in synchrony with the disassembly and reassembly of hNopp140 (originally named as p130) [28]. Using pol I as a 
marker for NORs in mitotic cells, we observed that the dispersed hNopp140, although not yet returned to NORs at anaphase, moved to chromosome surfaces containing pol I at telophase (Fig. 1a, upper panel; arrows for anaphase, arrowheads for telophase). Throughout interphase, hNopp140 colocalized with pol I in the nucleolar dot-like structures (Fig. 1a, lower panel). We then applied fluorescent in situ hybridization (FISH) with a probe encompassing the rDNA promoter region from -321 to -22 to locate rDNA clusters. Based on metaphase chromosome spread, this probe detected the short arms of acrocentric chromosomes, which are known with rDNA sequences (data not shown). In interphase, rDNA overlapped with hNopp140 in the nucleolar region (Fig. 1b); however, the extra-nucleolar hNopp140-containing spot (see arrow), likely as the Cajal body [37], was devoid of rDNA.
Previously we have shown that overexpressed hNopp140 can be effectively incorporated into a large sphere that expands the nucleolar region [33]. We therefore traced the rDNA by FISH of HeLa cells transfected with Flag-tagged hNopp140. Levels of ectopic expression of hNopp140 were monitored by anti-Flag antibody. Transfectants with increased expression of hNopp140 showed that rDNA repeats extended outwardly to the periphery of the enlarged sphere (Fig. 1c). Meanwhile, the peri-nucleolar condensed chromatin seemed also to extend to the same position. Note that in experiments using biotin-labeled probes, the endogenous biotin in the cytoplasm yielded a background staining that could define the nuclear-cytoplasmic boundary. Our results implied that the multiple hNopp140-containing dots could be merged into a single large sphere after incorporating the excessive hNopp140 at chromatin sites corresponding to the rDNA repeats.
Fig. 1 Colocalization of hNopp140, pol I and rDNA repeats. (a) Distribution of hNopp140 and pol I. The rounded-up mitotic cells were collected from HeLa culture by mechanical shake-off (upper panel; arrows for anaphase, arrowheads for telophase). The attached cells were at interphase (lower panel). Cells were subjected to double-fluorescent immunostaining of pol I and hNopp140. The DNA was counterstained with Hoechst 33258. (b) Distribution of hNopp140 and rDNA. HeLa cells at interphase were visualized in FISH experiments with a biotin labeled rDNA probe and a detector, avidinrhodamin conjugate. The same cells were also

immunofluorescence-stained with anti-hNopp140 mAb. Arrow, Cajal body. (c) rDNA colocalized with the ectopically expressed hNopp140. HeLa cells transfected with Flagtagged full-length hNopp140 were processed for detecting the rDNA and the Flag-tagged proteins. Bars, $10 \mu \mathrm{m}$

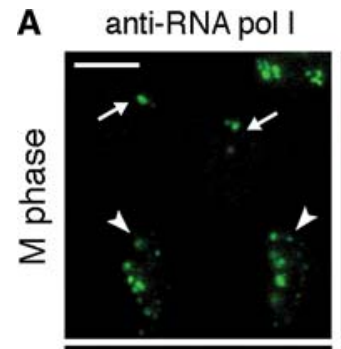

anti-hNopp140
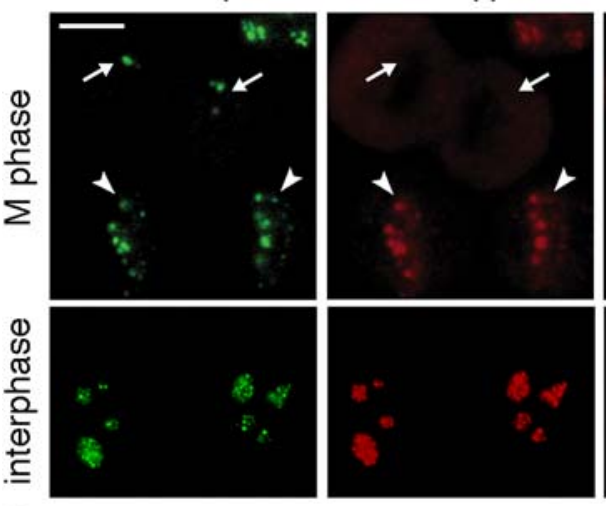

B anti-hNopp 140

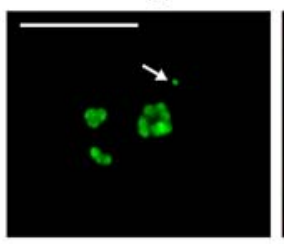

rDNA

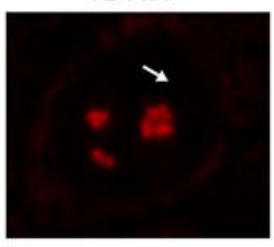

merged
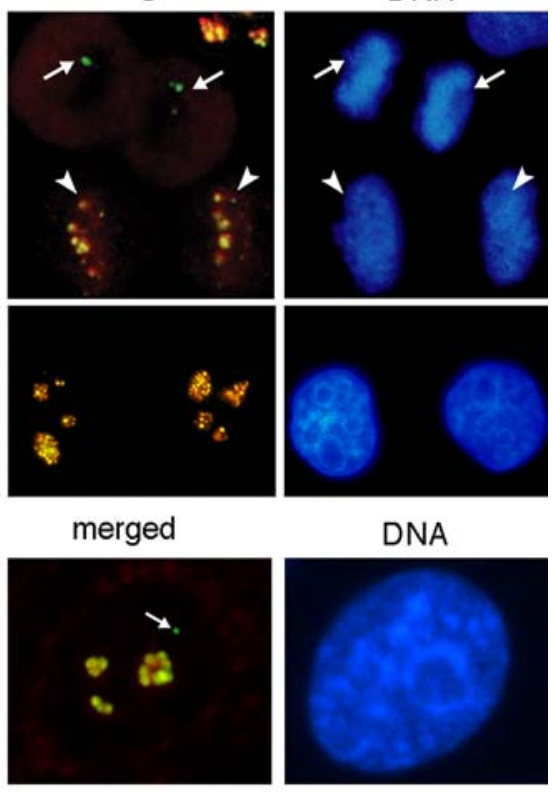

C F-hNopp140 transfection
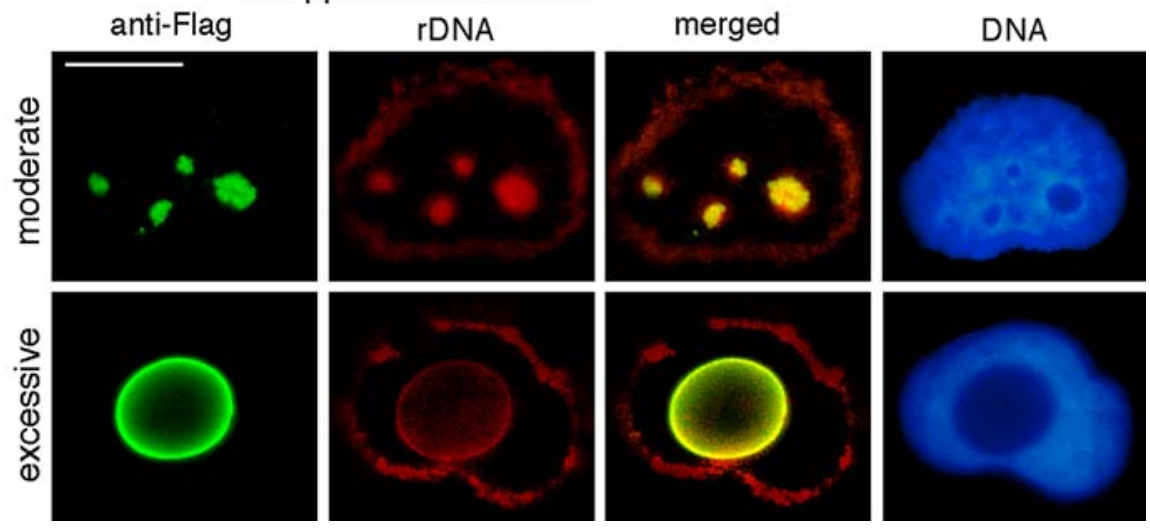
Effects of the conserved N- and C-termini of hNopp140 on the localization of rDNA repeats

The DM1 of hNopp140 represents a mutant missing the conserved C-terminal domain (Fig. 2a). Unlike the fulllength hNopp140, it retained less effectively in the nucleolus, as indicated by immunofluorescence in the nucleoplasm. Strikingly, it always formed sphere-like entities in nucleoli with invaginations at one or two poles (Fig. 2b; see also Fig. 3c, g and h). Interestingly, the rDNA was largely accumulated in the indent areas. There were different distribution patterns of the rDNA. In one form, the rDNA repeats began to withdraw from the DM1-containing spheres (Fig. 2b, i, arrows; note that the red area is smaller than the green area, but they are still superimposed). Others showed that the rDNA repeats were separated from the DM1 to display the hat-shaped (Fig. 2b, ii and iii, arrowheads) and the compact (Fig. 2b, i, iii and iv, dashed arrows) patterns of distribution. The rDNA tended to be free from DM1, indicating that the C-terminal domain of hNopp140 might be involved in interaction with a chromatin site proximal to the rDNA locus.

We next examined the phenotypes of DM2 transfectants that expressed a mutant hNopp140 lacking its conserved Nterminal domain. When expression levels were low, DM2 formed round-shaped structures whose peripheries displayed an uneven distribution of DM2. The rDNA repeats localized in the DM2-rich area (Fig. 2c, i). Some exhibited patched patterns of DM2 distribution, and, notably, rDNA repeats always colocalized with DM2 (Fig. 2c, iii and iv, arrows). As the DM2 expression increased, the small circles coalesced into large asymmetric spheres that harbored rDNA repeats wherever DM2 was abundant (Fig. 2c, ii). Effects of DM2 were completely opposite from those of DM1.

The DM3 with only the central ten repeats of hNopp 140 (Fig. 2a) was able to target to the nucleolus, but neither

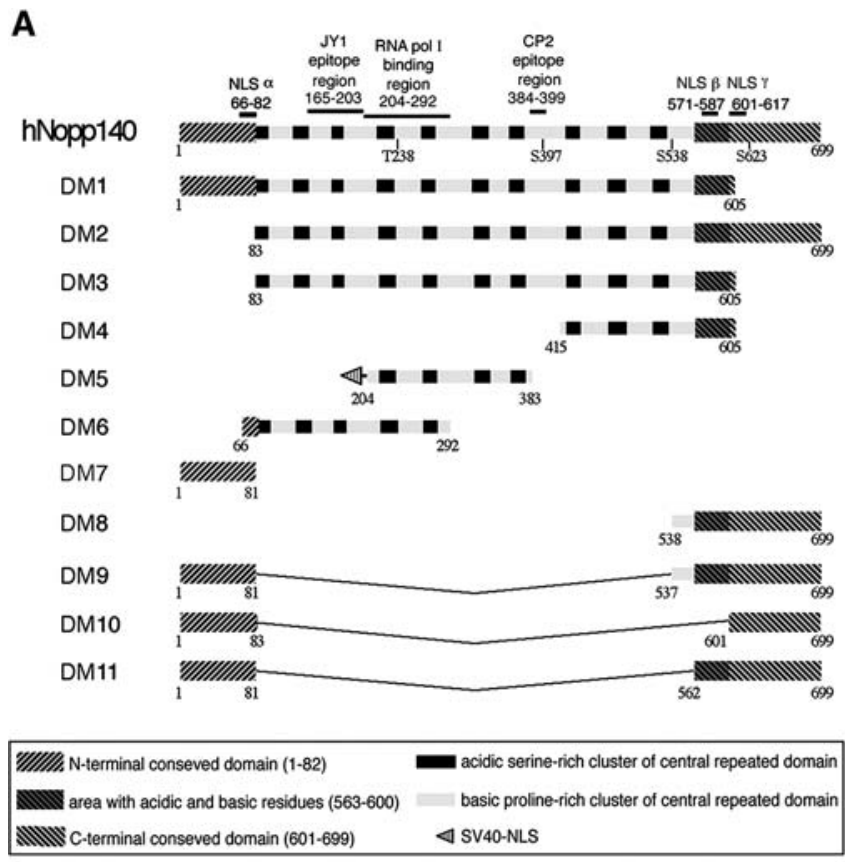

B F-DM1 transfection

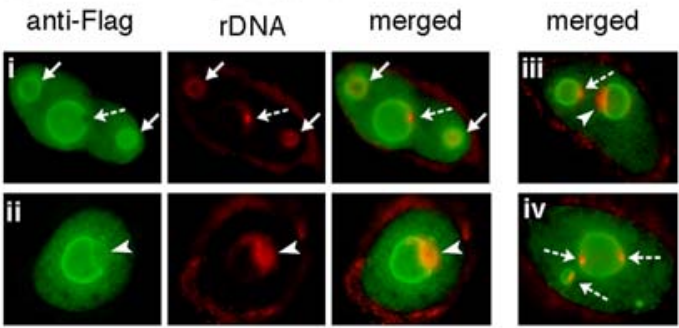

C F-DM2 transfection

anti-Flag
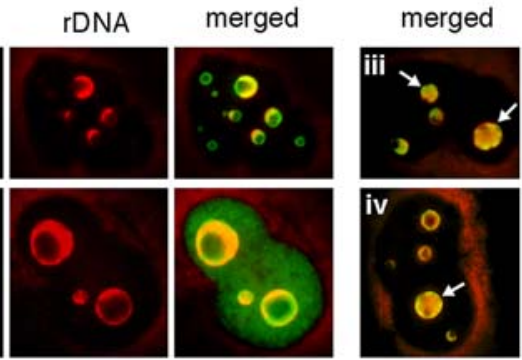

D F-DM3 transfection anti-Flag
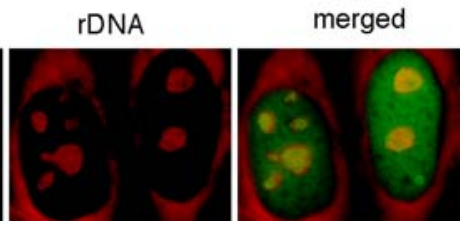

Fig. 2 Mislocalization of rDNA repeats induced by hNopp140 mutants. (a) Schematic representation of the wild and mutant types of hNopp140. Numbering refers to the amino acid residues on hNopp140. Three NLS, four CDK1/cyclin B phosphorylation sites (T238, S397, S538, S623), pol I binding region, and epitope regions recognized by mAbs CP2 and JY1 are indicated. (b-d) HeLa cells were transfected with Flag-tagged hNopp140 mutant constructs DM1, DM2 or DM3. After 48 h, FISH experiments to detect rDNA repeats combined with anti-Flag immunofluorescence staining were performed. (b) Segregation of the rDNA from the DM1-containing spheres. Arrows, arrowheads and dashed arrows stand for positional changes of the rDNA at three different stages as described in the text. Panels iii and iv display the merged images only. (c) The rDNA shifted to the DM2-rich region. Arrows indicate the spherical structure with unevenly distributed DM2 on the surface domain resulting in a patched pattern, which is distinct from the majority with DM2 unequally arranged at the periphery. The rDNA always colocalizes with DM2. Panels iii and iv show the merged images only. (d) Effects of DM3 on the rDNA distribution. DM3 targeted to the nucleolus without altering the rDNA localization. Bar, $10 \mu \mathrm{m}$ 
Fig. 3 Acrocentric $\alpha$-satellite arrays, pol I, UBF and rDNA transcription affected by hNopp140 mutants. (a and f) Normal controls. Normally growing HeLa cells were immunostained with antihNopp140, combined with FISH using a probe specific to the $\alpha 13,14,21$ (a), or the $\alpha 9$ (f). (b-e, $\mathbf{g}$ and $\mathbf{h}$ ) Transfection experiments. HeLa cells were transfected with Flag-tagged full-length hNopp140, or deletion mutants DM1, DM2 and DM3 as indicated. After $48 \mathrm{~h}$, fluorescent staining was performed to detect the ectopically expressed proteins by anti-Flag and the endogenous pol I or UBF by specific mAbs (g), or to detect Flag-tagged proteins combined with FISH to locate the $\alpha 13,14,21$ (b-e).

Panels $\mathbf{c}$ ii and $\mathbf{d}$ ii display the merged images only. In one set of the $48 \mathrm{~h}$ transfection experiments, the DM1 and DM2 transfectants were

permeabilized for Br-UTP incorporation (h). The nascent Br-UTP labeled RNA (a 10 min pulse-labeling) was detected by anti-Br-UTP. Meanwhile, the DM1, DM2 and the endogenous hNopp140 were detected by anti-hNopp140 mAb, CP2. Bars, $10 \mu \mathrm{m}$

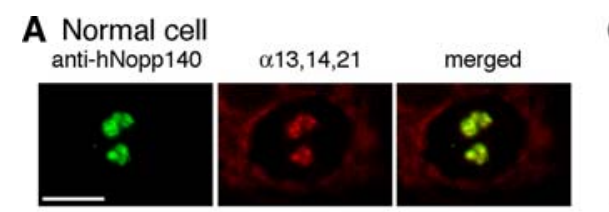

G endogenous RNA pol I and UBF
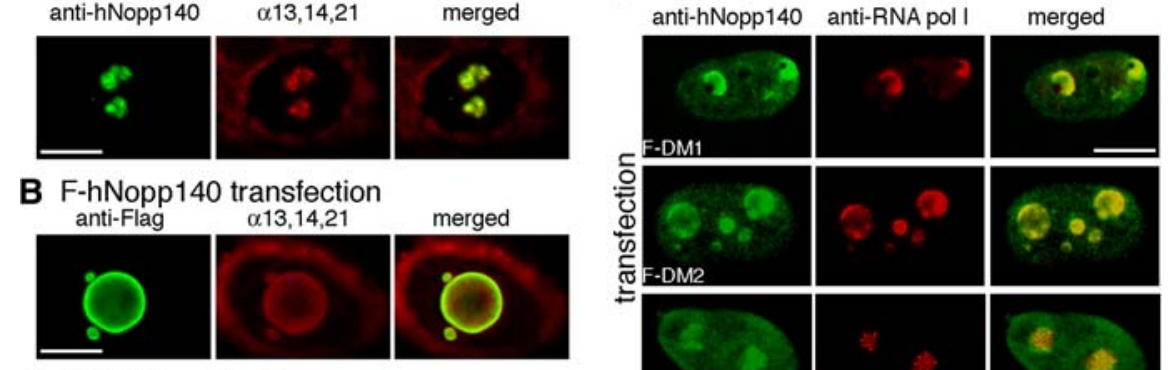

C F-DM1 transfection
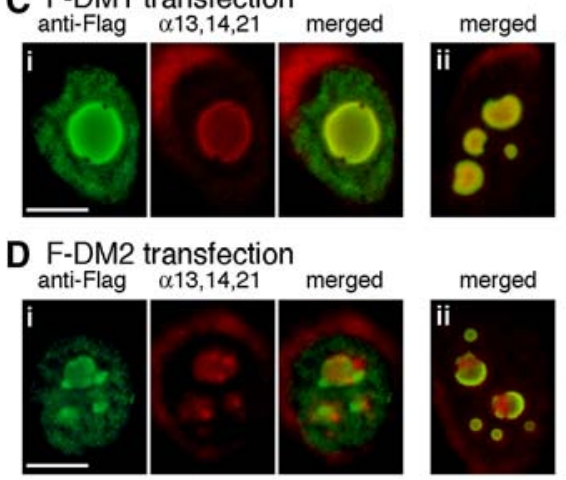

E F-DM3 transfection

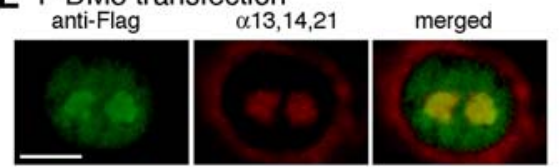

F $\alpha$-satellite control

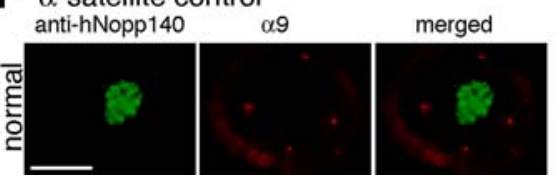

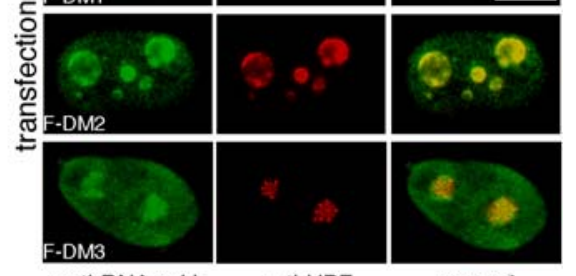

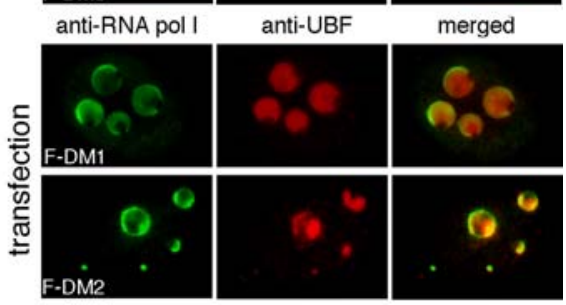

H in situ run-on transcription assay

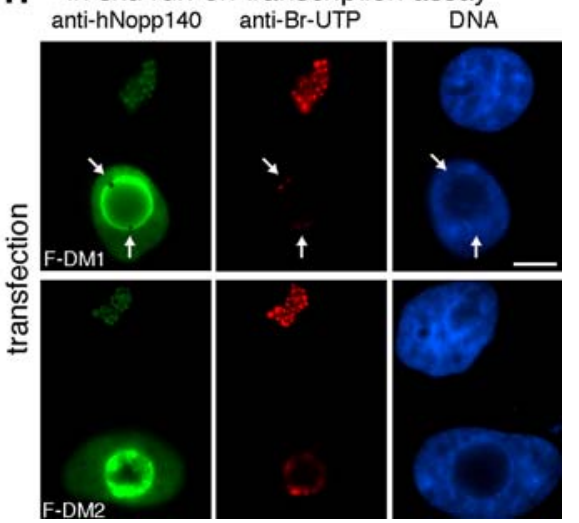

affected the distribution of rDNA, nor altered the general nucleolar appearance (Fig. 2d; see also Fig. 3g). The maximal intensity of DM3 in nucleoli could reach a constant, and the excessive DM3 was accumulated in the nucleoplasm (Fig. 2d, compare the two cells; see also Fig. 3e and g). Results derived from DM1, DM2 and DM3 support that the $\mathrm{N}$ - and the C-termini of hNopp140 work differently in interactions with nucleolar chromatin.

Acrocentric $\alpha$-satellite sequences are involved in the hNopp140-mediated nucleolar topographic organization

Evidence has shown that centromeric DNA sequences, as detected in situ by a probe to all-human-centromere $\alpha$ satellite, locate preferentially around the nucleolus and at the nuclear periphery [44]. Consistently, autoantibodies to centromeric proteins detect interphase centromeres in locations around and within nucleoli as well as along the nuclear envelope [45]. Recent data have demonstrated that nucleolar tau interacts with AT-rich pericentromeric satellite sequences [46]. Thus, there are links between nucleolus and centromeric heterochromatin. We observed that overexpressed hNopp140 remained at sites corresponding to the peri-nucleolar condensed chromatin (Fig. 1c), which is known to have centromeric sequences [13-15]. Although the C-terminally truncated mutant of hNopp140 lost the ability to colocalize with rDNA, it still anchored at positions equivalent to the extended perinucleolar chromatin (Fig. 2b). Apparently, there could be chromatin-binding sites distal to rDNA loci for retention of this mutant protein in the nucleolus. We, therefore, used a 287 bp $\alpha$-satellite DNA probe, which selectively detects acrocentric chromosomes 13, 14 and 21 [7], to monitor the locations of these $\alpha$-satellite elements in control and transfected HeLa cells. The validity of the probe was demonstrated on metaphase spreads, of which only regions near the ends representing the centromeres of the acrocentric chromosomes were hybridized (data not shown). In interphase, the hNopp140-containing islets were embedded within areas positive to the $\alpha$-satellite of chromosomes 13 , 
14 and $21(\alpha 13,14,21)$ in the control HeLa cells (Fig. 3a). In cells overexpressing hNopp140, the $\alpha 13,14,21$ arrays were enriched at the periphery of the enlarged sphere (Fig. 3b), a pattern similar to that of rDNA (Fig. 1c). Note that the adjacent small circles before integrated into the large sphere were composed of both the hNopp140 and the $\alpha 13,14,21$. A negative control probe, which is specific to the centromeric $\alpha$-satellite elements of chromosome $9(\alpha 9)$ [40], did not hybridize the chromatin at the nucleolar region (Fig. 3f).

In mutant expression experiments, the acrocentric $\alpha$ satellite arrays colocalized with DM1 in a concave pattern without occupying the indent spaces (Fig. 3c), but filled up areas of the sphere wherever DM2 was sparse (Fig. 3d). Thus, the $\alpha 13,14,21$ became separate from the rDNA in both DM1 and DM2 transfectants (compare Fig. 3c and d with Fig. 2b and c, respectively). Disruption of the nucleolar structure was further supported by the evidence that pol I was mislocalized to the DM1 and DM2 rich regions (Fig. 3g, the first and second rows). In contrast, DM3 did not alter distribution of the $\alpha 13,14,21$ (Fig. 3e). The normally nucleolar punctate pattern of pol I also supported that DM3 did not change the general nucleolar architecture (Fig. 3g, the third row). Furthermore, double immunofluorescence staining for detecting the endogenous pol I and upstream binding factor (UBF) demonstrated that these two proteins were colocalized in the altered nucleoli where the DM1 or the DM2 was also accumulated as judged by the distinct phenotypes of cells expressing these two types of truncated hNopp140 (Fig. $3 \mathrm{~g}$, the fourth and fifth rows).

We also analyzed rDNA transcription in cells expressing DM1 or DM2. A faint incorporation of Br-UTP occurred at the border of the invaginations of DM1-containing spheres (Fig. 3h, upper row; arrows point out the invaginations). It was already known that in these DM1-derived structures, the rDNA was condensed mostly in the indent areas (Fig. 2b), where pol I and UBF were excluded (Fig. 3g). Thus, residual levels of rDNA transcription took place only at the contact sites between rDNA and transcription machinery. On the other hand, Br-UTP incorporation was detected at sites of DM2-enriched region (Fig. 3h, lower row), where the rDNA, pol I, and UBF were collected together (Figs. 2c and 3g). We have constantly observed that the inhibition of rDNA transcription was more severe in the altered nucleoli induced by DM1 than those by DM2 (compare the transfected cells with the neighboring untransfected cells in Fig. 3h). All results argue for that the $\mathrm{N}$ - and C-termini of hNopp140 interplay respectively with chromatin regions containing the $\alpha$-satellite and rDNA repeats of acrocentric chromosomes during interphase for the correct nucleolar assembly and the optimal rDNA transcription.
The joined N- and C-terminal domains of hNopp140 anchor on the nucleolar condensed chromatin

In order to further evaluate roles of the two ends of the hNopp140 molecule, we constructed deletion clones encoding the N-terminal domain (DM7), C-terminal domain (DM8; NLS- $\beta$ region also included for a better nuclear targeting), and fused $\mathrm{N}$ - and $\mathrm{C}$-terminal domains (DM9) of hNopp140 (Fig. 2a). Transfectants expressing the Flag-tagged DM7, DM8 or DM9 were subjected to double immunofluorescence staining with mAbs to Flagtag and to hNopp140 (amino acids 384-399 as the epitope region). Thus, localization of the mutant proteins and the endogenous hNopp 140 could be traced simultaneously. We observed that at low expression levels, all DM9 targeted to nucleolus and colocalized with the endogenous hNopp140 (Fig. 4b, dashed arrows). As the expression levels increased, DM9 frequently showed a ring-like distribution pattern (Fig. 4a and b, arrows), which matched well with the peri-nucleolar condensed chromatin (Fig. 4a, right panel, arrows). The rings became masked when the nucleoplasm collected more DM9. Nevertheless, DM7 or DM8 did not form distinct rings (Fig. 4c and d). Occasionally very faintly stained rings were seen. At much higher expression levels, all showed relatively homogeneous distribution patterns in nuclei (Fig. 4b-d, left panels, arrowheads); however, only DM9 (no longer with visible rings), but not DM7 and DM8, caused the endogenous hNopp140-containing dots to be spread into nucleoplasm (Fig. 4b-d, arrowheads). This phenomenon occurred only when cells overexpressed DM9 or two other similar mutant proteins, DM10 and DM11 (Fig. 2a), demonstrating the competition between the endogenous hNopp140 and the mutant proteins for the same binding sites.

Great overexpression of the joined N- and C-terminal domains of hNopp140 disrupts the nucleolar structure and induces global chromatin condensation

We unexpectedly found that greatly overexpressed DM9 induced dramatic changes for not only the nucleolar structure but also the general chromatin appearance. Cells with global chromatin condensation were immediately noticed after DNA staining, and such patterns matched well with the great overexpression of DM9 (Fig. 5a and b, compare DNA staining patterns with anti-Flag intensities). Cells with the DM9-induced spreading of the hNopp140containing dots into the entire nucleoplasm had not yet displayed chromatin condensation (Fig. 5a, see arrowheads in the first row). As the DM9 expression was increased further, chromatin condensation then became obvious, which was always accompanied with phenomena that 


\section{A F-DM9 transfection}

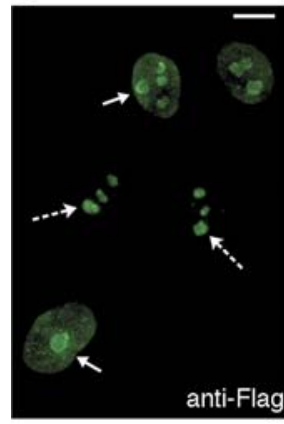

C

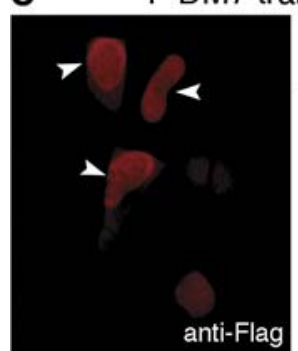

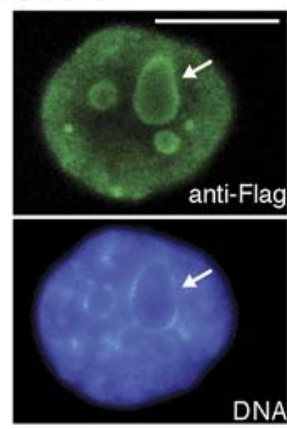

DNA

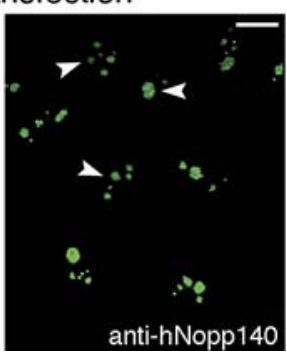

Fig. 4 Analysis of the N- and C-terminal parts of hNopp140. HeLa cells were transfected with Flag-tagged DM7 (c), DM8 (d) or DM9 (a and b). After $48 \mathrm{~h}$, the mutant proteins and the endogenous hNopp 140 were visualized by double-immunofluorescent staining with anti-Flag and anti-hNopp140 mAbs. Cells expressing medium levels of DM9 showed a ring-like pattern at the peri-nucleolar condensed chromatin region (arrows in $\mathbf{a}$ and $\mathbf{b}$ ); under such conditions, DM9 did not cause the dispersion of the endogenous hNopp140 (arrows in b). In cells

hNopp140 and UBF were undetectable or fully dispersed, and that pol I was densely packed (Fig. 5a, arrows; see also Fig. 5b). FISH experiments demonstrated that as long as cells underwent global chromatin condensation, their rDNA repeats were transformed into a compact form at sites where pol I was also concentrated (Fig. 5b, the first and the third rows, see arrows). In contrast, the $\alpha 13,14,21$ arrays were dispersed away and became hardly detectable in cells with condensed chromatin due to extremely high expression of DM9 (Fig. 5b, the second row, arrows). Note that one of such cells showed that the $\alpha 13,14,21$, at a marginally detectable level, was segregated from the compactly stored pol I (Fig. 5b, the fourth row, arrows). As a control, the adjacent cells with the normal morphology exhibited pol I being colocalized with the $\alpha 13,14,21$. Therefore, DM9, functioning as an anchoring domain, possesses certain abilities to influence the nucleolar structural organization and the chromatin topology.

Acrocentric $\alpha$-satellite and rDNA sequences recognized respectively by the $\mathrm{N}$ - and $\mathrm{C}$-terminal domains of hNopp 140

In order to confirm that hNopp140 can provide a matrix environment for tethering the rDNA repeats and the

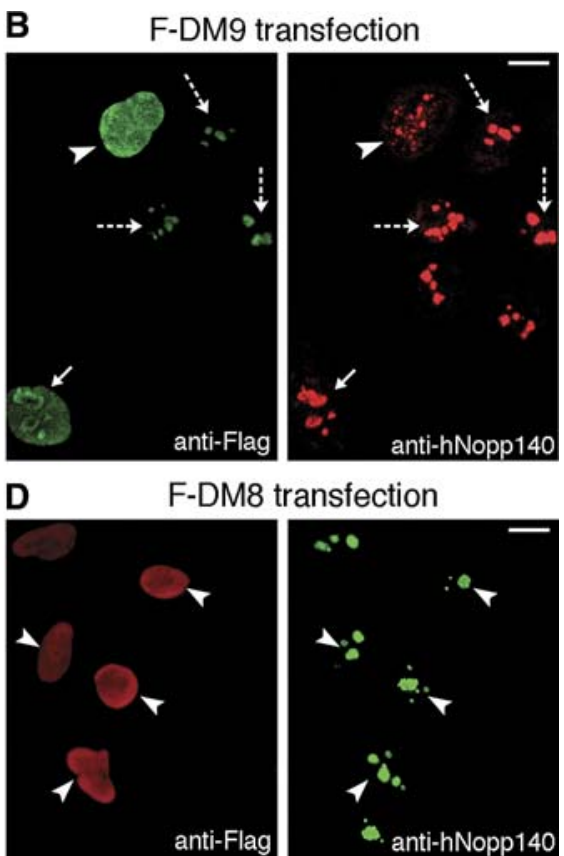

with much higher expression of DM9, the ring became masked and the endogenous hNopp140 was spread into nucleoplasm (arrowheads in b). At low expression levels, all DM9 appeared in nucleolus (dashed arrows in $\mathbf{a}$ and $\mathbf{b}$ ) and colocalized with the endogenous hNopp140 (dashed arrows in b). DM7 and DM8 were distributed homogeneously in the nucleus and did not affect the endogenous hNopp140 (arrowheads in c and d). Bars, $10 \mu \mathrm{m}$

acrocentric $\alpha$-satellite arrays, we performed ChIP assays using anti-hNopp140 antibody to pull down the bound chromatin, and PCR primer sets to amplify sequences of the rDNA promoter, $\alpha 13,14,21$ and $\alpha 9$. Both the rDNA and $\alpha 13,14,21$, but not the negative control $\alpha 9$, were present in the hNopp140-immunoprecipitates prepared from HeLa cell lysates (Fig. 6a). We then analyzed whether the rDNA and the acrocentric $\alpha$-satellite repeats could be co-immunoprecipitated by ectopically expressed DM9, a potential anchoring domain of hNopp140 that can target to nucleolus and compete off the endogenous hNopp140. As expected, this joined $\mathrm{N}$ - and C-terminal domains of hNopp140 was effectively cross-linked to both the rDNA and the $\alpha 13,14,21$ (Fig. 6b, lanes 2-5). Using untransfected cells as a negative control, the anti-Flag antibody precipitated neither the rDNA nor the $\alpha 13,14,21$ repeats (Fig. 6b, lanes 6-9).

Classical EMSA using biotin-labeled probes of rDNA (300 bp) or acrocentric $\alpha$-satellite DNA (287 bp) was established to test the DNA binding abilities of hNopp140. Immunopurified hNopp140 and its C-terminal domain (DM8) showed retardation effects on the rDNA probe; these interactions were competed out by addition of excessive unlabeled rDNA probe (Fig. 6c). As expected, UBF also bound specifically to this rDNA promoter region (Fig. 6c, lanes 2-5). Importantly, the N-terminal domain of 
Fig. 5 Global chromatin condensation and nucleolar disruption induced by the joined $\mathrm{N}$ - and C-terminal parts of hNopp140. HeLa cells were transfected with Flag-tagged DM9. After $48 \mathrm{~h}$, two-color fluorescent staining was performed to detect either two proteins as indicated in (a), or one protein combined with FISH to locate rDNA or $\alpha 13,14,21$ as shown in (b). The chromatin distribution patterns were revealed by Hoechst 33258 staining. Cells with condensed chromatin (marked by arrows) correlated well with the great overexpression of DM9. They showed the compact and brightly stained patterns for pol I and rDNA. On the contrary, the dimly stained and dispersed patterns were seen for hNopp140, UBF and $\alpha 13,14,21$. Note that cells with the hNopp140-containing dots spread over nucleoplasm (see arrowheads in the upper row of panel a) were apparently at a stage before the chromatin condensation commenced. Merged images are shown on the right. Within the same microscopic fields, cells not transfected, or with DM9 expression at low levels, can serve as the control that exhibit normal distributions of nucleolar proteins, rDNA and $\alpha 13,14,21$. Bar, $10 \mu \mathrm{m}$

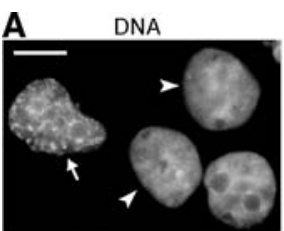

DNA

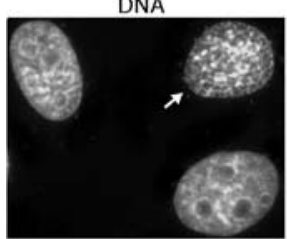

DNA

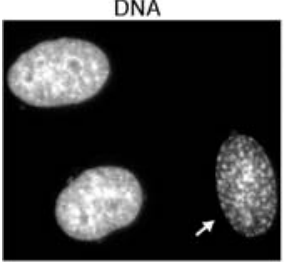

B

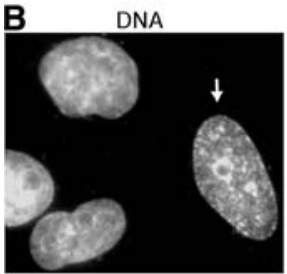

DNA

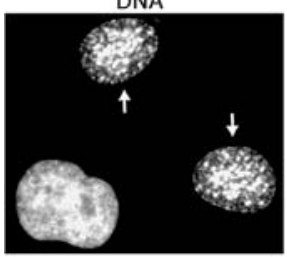

DNA

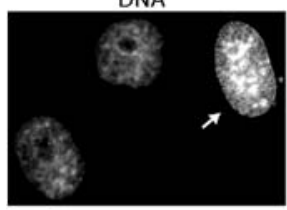

DNA

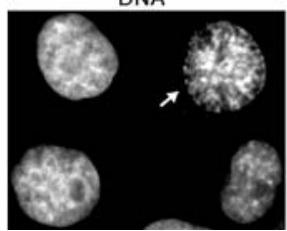

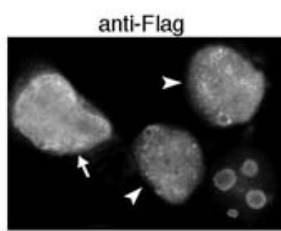

anti-Flag

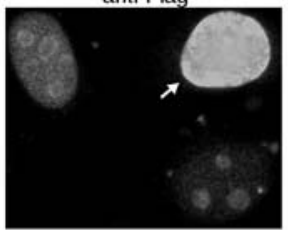

anti-RNA pol I

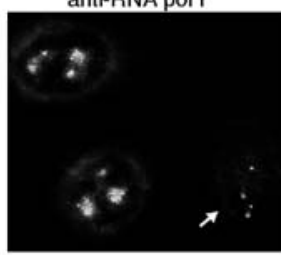

anti-Flag

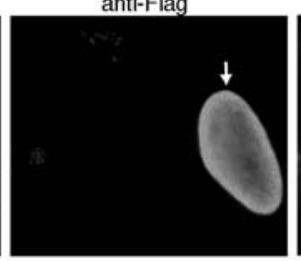

anti-Flag

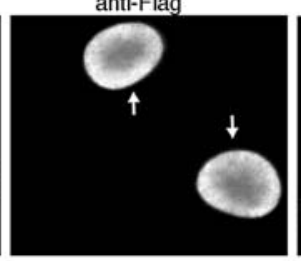

anti-RNA pol I

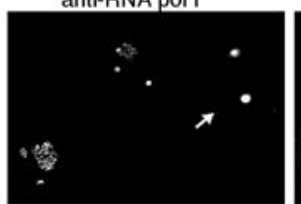

anti-RNA pol I

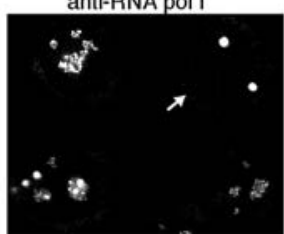

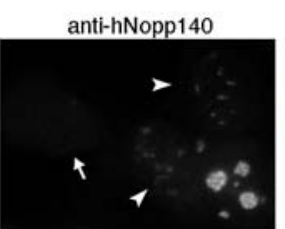

anti-RNA pol I

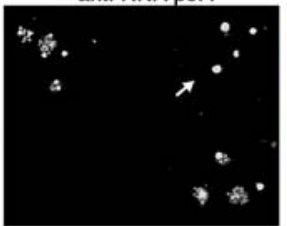

anti-UBF

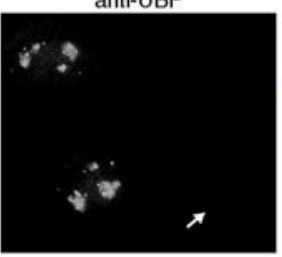

rDNA

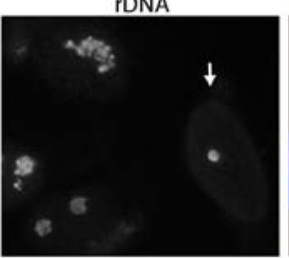

$\alpha 13,14,21$

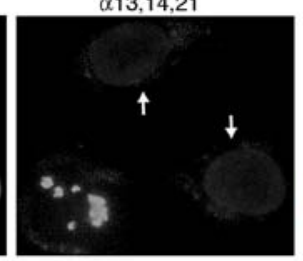

rDNA

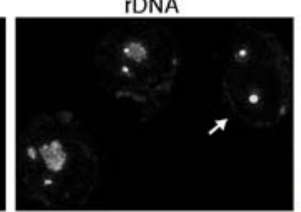

$\alpha 13,14,21$

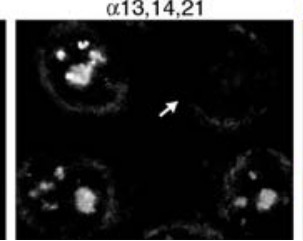

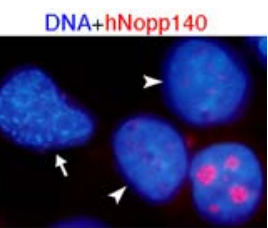

DNA+RNA pol I

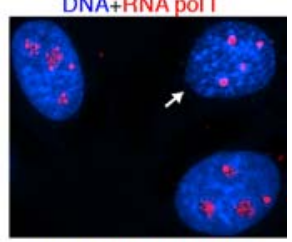

DNA+RNA pol I+UBF

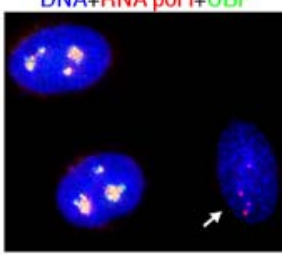

DNA+rDNA

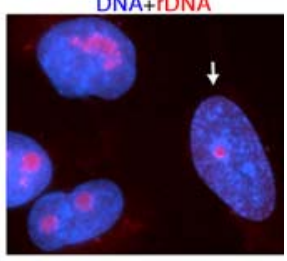

DNA $+\propto 13,14,21$

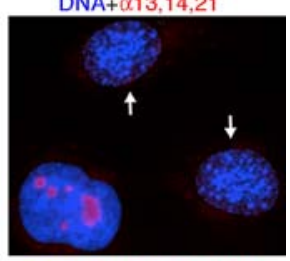

DNA+RNA pol I+rDNA

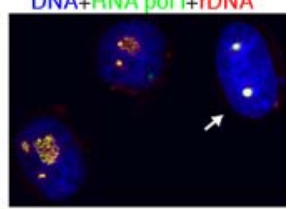

DNA+RNA pol $++\alpha 13,14,21$

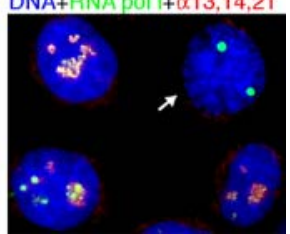

hNopp140 (DM7) exhibited no binding at all to the rDNA probe (Fig. 6c, lane 16). A similar experimental design demonstrated that hNopp140 and its $\mathrm{N}-$, but not C-, terminal domain interacted specifically with the $\alpha$-satellite probe (Fig. 6d). Interestingly, the acrocentric $\alpha$-satellite probe bound also to the immunopurified tau (Fig. 6d, lanes 2-5), which is known to interact with AT-rich satellite sequences [46]. These gel shift data have confirmed that the $\mathrm{N}$ - and C-terminal domains of hNopp140 are for tethering the acrocentric $\alpha$-satellite and rDNA sequences, respectively.

The central ten repeats of hNopp140 display a weak self-self interacting ability

Overexpressed hNopp140 formed a large sphere in nucleolus, and therefore, in addition to the chromatin 
A anti-hNopp140 ChIP

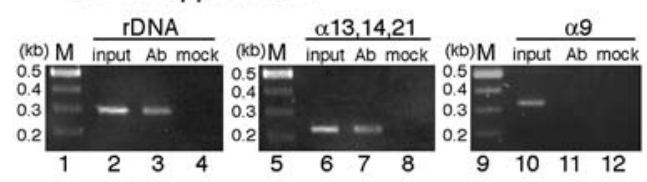

B transfection, anti-Flag ChIP

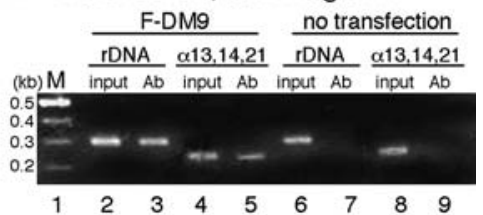

\section{EMSA: rDNA probe}
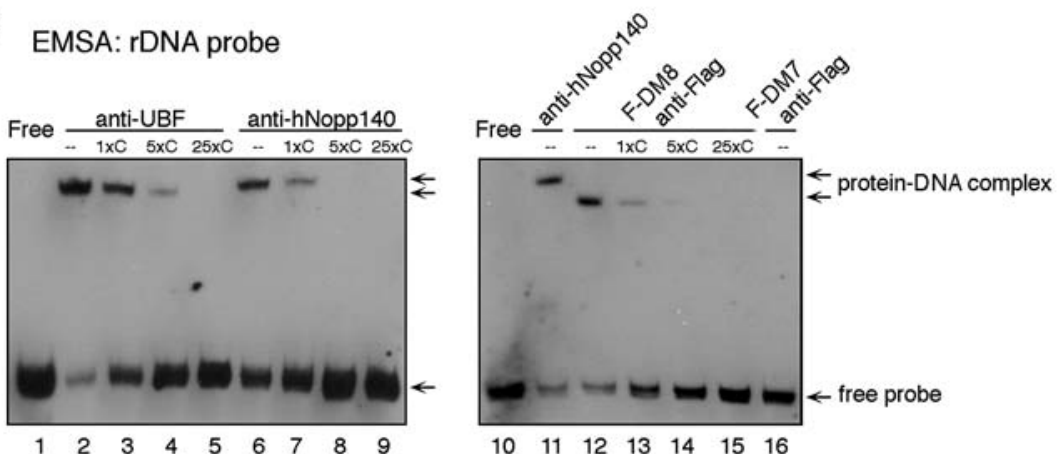

D

EMSA: $\alpha 13,14,21$ probe
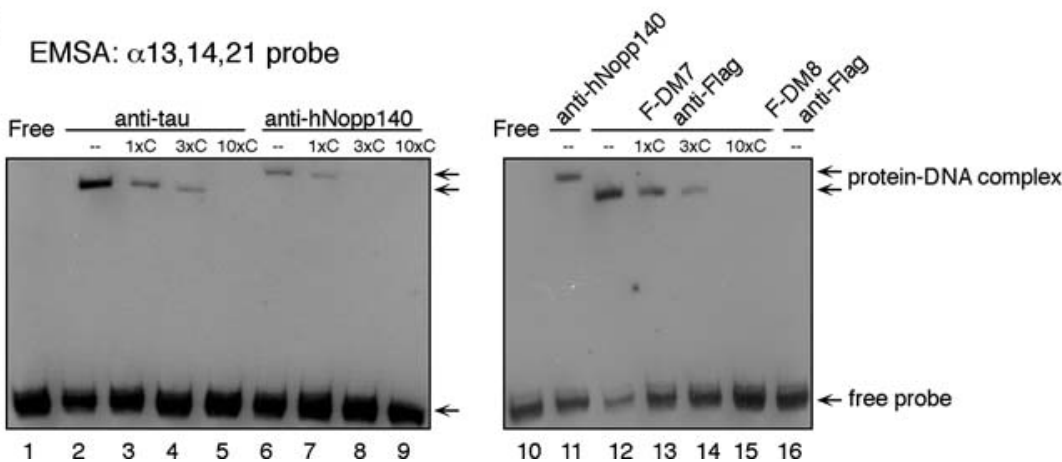

Fig. 6 Acrocentric $\alpha$-satellite and rDNA sequences recognized respectively by the N- and C-terminal domains of hNopp140. (a) Anti-hNopp140 ChIP assay. Cell lysates prepared from normally growing HeLa cells were processed for ChIP analysis for detecting rDNA, $\alpha 13,14,21$, and $\alpha 9$ in the anti-hNopp 140 pull-down complexes, or omitting antibody as the mock controls. M, DNA size markers. (b) Anti-Flag ChIP assay. The 293T cells were transfected with Flagtagged DM9. After $48 \mathrm{~h}$, ChIP analysis was performed to detect rDNA and $\alpha 13,14,21$ in the anti-Flag immunoprecipitates. Negative controls without transfection were included. (c) rDNA EMSA. Immunoprecipited proteins using antibodies to UBF (lanes 2-5),

recognition features as described above, the hNopp140 molecule should have at least one self-self interacting domain that could assist the formation of a spherical structure. To test this, we cotransfected the wild type of hNopp140 or its mutant DM3, encoding only the central ten repeats, each with two different tags, into $293 \mathrm{~T}$ cells. Specific interactions were examined when lysates were reciprocally precipitated with one tag-specific antibody and blotted with the other. Flag- and Myc-tagged hNopp140 were coimmunoprecipitated by each other (Fig. 7a). In contrast, the central ten repeats of hNopp140 showed a weak interaction (Fig. 7b). We further performed coimmunoprecipitation assays on cell lysates from 293T cells after transient transfection with a number of Flag-tagged deletion mutants of hNopp140. The endogenous hNopp140

hNopp140 (lanes 6-9, and 11), or Flag-tag (lanes 12-15 for DM8 transfection; lane 16 for DM7 transfection) were incubated with biotin-labeled rDNA probe. For competition, unlabled rDNA probe at $1-, 5-$, or 25-fold excess was added in the beginning of the binding reaction. Arrows indicate positions of the free probe and the proteinDNA complex. (d) Acrocentric $\alpha$-satellite EMSA. Experimental design was similar to that of (c). Immunoprecipited proteins using antibodies to tau, hNopp140, or Flag-tag (for DM7 or DM8 transfection) were incubated with biotin-labeled $\alpha 13,14,21$ probe in the absence or presence of unlabled $\alpha 13,14,21$ (competitor) at 1-, 3-, or 10 -fold excess

was examined in the complexes when lysates were precipitated with anti-Flag and blotted with anti-hNopp140 (Fig. 7c). As expected, the central ten repeats of hNopp140, DM3, showed only a low potency to precipitate the endogenous hNopp140 (Fig. 7c, lane 3). However, DM4, DM5 and DM6, representing various parts of the central repeated domain of hNopp140 (Fig. 2a), completely lost the ability to precipitate hNopp140 (Fig. 7c, lanes 12, 9 and 6). Surprisingly, mutants with the joined $\mathrm{N}$ and C-terminal domains of hNopp140 (DM9 or DM10) precipitated the endogenous hNopp140 more effectively than the DM3 did (Fig. 7c, lanes 15 and 18).

We suspected that chromatin with rDNA repeats or acrocentric $\alpha$-satellite arrays might bridge the DM9 (or DM10) together with the endogenous hNopp140 in 


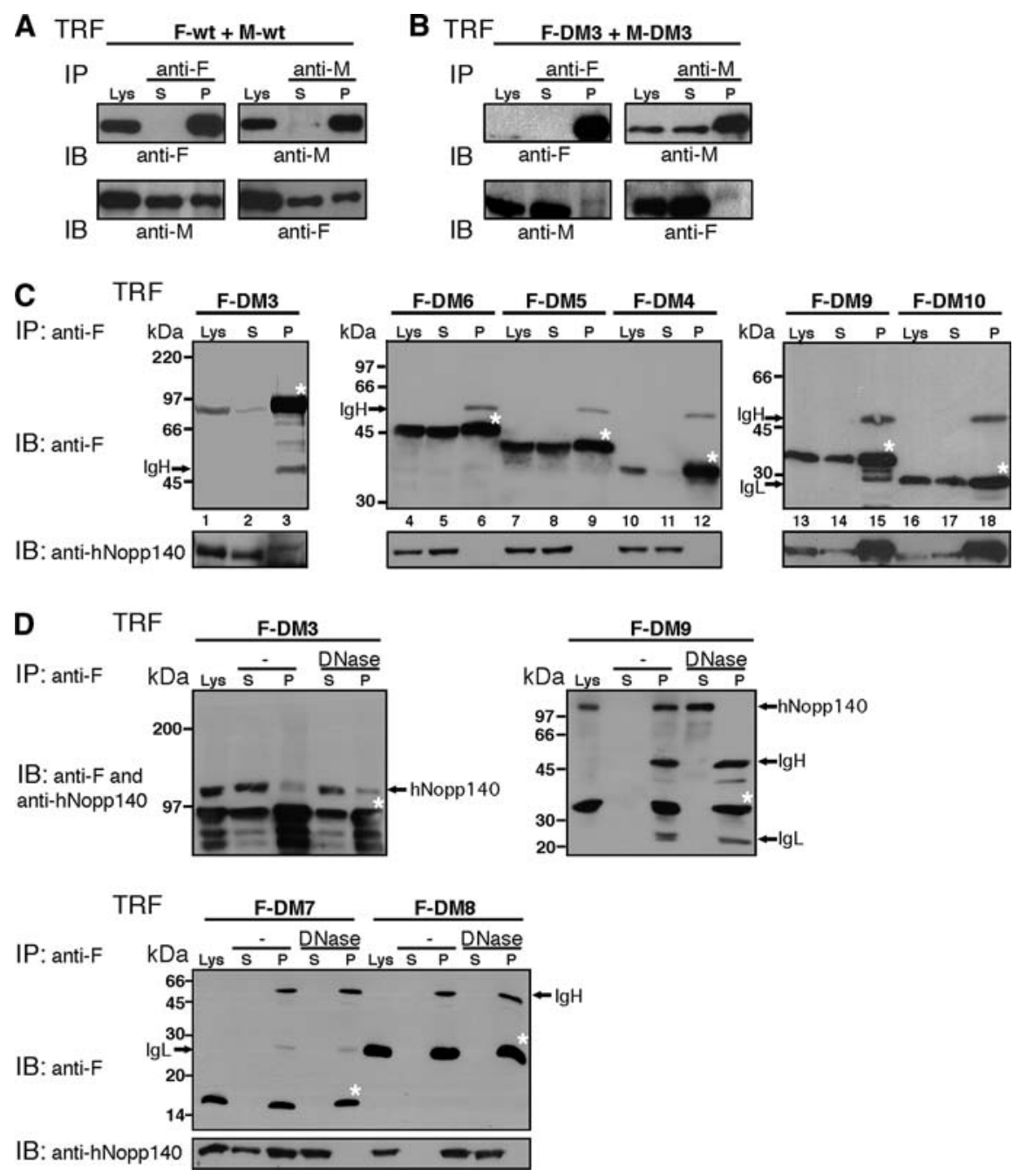

Fig. 7 Determination of the self-self interacting domain of hNopp140. (a and b) 293T cells were cotransfected with Flag- [F-] and Myc- [M-] tagged wild type [wt], or a mutant type DM3, of hNopp140. After $44 \mathrm{~h}$, reciprocal immunoprecipitation assays were performed. The input cell lysates [Lys], or the supernatants [S] and pellets $[\mathrm{P}]$ after immunoprecipitation, were analyzed by immunoblotting with the indicated antibodies. No bands appeared when omitting the antibody during the immunoprecipitation (not shown). (c and d) Lysates from 293T cells transfected individually with Flag-tagged

co-immunoprecipitation experiments, a situation already hinted by the ChIP assays (Fig. 6b). This turned out to be true, since DNase digestion of the anti-Flag immunoprecipitates, prepared from DM9-transfected 293T cells, allowed the bound endogenous hNopp140 to be released into the supernatant (Fig. 7d, upper right panel). Similar results were obtained when we examined whether the endogenous hNopp140 interacts with fragments containing its own $\mathrm{N}$ - or C-terminal domains, as represented by DM7 and DM8, respectively (Fig. 7d, lower panel). However, the same DNase treatment did not alter the weak interaction between DM3 and the endogenous hNopp140 (Fig. 7d, upper left panel). Thus, the homologous self-self mutant constructs of hNopp140 as indicated were immunoprecipitated by anti-Flag. For one set of samples in (d), DNase was added to the lysate mixed with the antibody-coated beads during the overnight tumbling at $4^{\circ} \mathrm{C}$. Immunoblot analysis was conducted using anti-Flag and anti-hNopp140. The asterisks mark the deletion mutants with expected molecular masses. Degraded forms of DM3 with smaller sizes were also detected. TRF, transfection; IP, immunoprecipitation; IB, immunoblotting; IgL, Ig light chain; IgH, Ig heavy chain

interacting domain is contributed by the central ten repeats of hNopp140.

Knockdown of hNopp140 results in the dispersion of rDNA and $\alpha 13,14,21$ and the arrest of pre-rRNA synthesis

Knockdown of the endogenous hNopp140 by RNA interference was also performed. HeLa cells were transiently transfected with specific siRNA duplexes by using lipofectamine. After incubation for $72 \mathrm{~h}$, about $70 \%$ of the cell populations, as estimated at the single cell level, 
showed down-regulation of hNopp140. Thus, the neighboring cells either with or without reduction of the endogenous hNopp140 could be compared with each other. Localizations of UBF and pol I were not noticeably affected when the hNopp140 was reduced to a barely detectable level (Fig. 8a and b). In contrast, the rDNA and $\alpha 13,14,21$ repeats were dispersed away from the nucleoli after RNAi knockdown of hNopp140 (Fig. 8c and $\mathrm{d}$; note that the signals for $\alpha 13,14,21$ can be seen in the nucleoplasm). Additionally, using a non-targeting siRNA as the control, the normal distributions of rDNA and $\alpha 13,14,21$ were observed (data not shown). rDNA transcription was then assessed by monitoring Br-UTP incorporation into nascent nucleolar RNA. Evidently, the nucleolar uptake of Br-UTP was decreased to a near undetectable level in cells with hNopp140 being effectively down regulated (Fig. 8e). Because the rDNA transcription machinery remained in the nucleolus under the same situation (Fig. 8a and b), transcriptional silence was likely due to the mislocalization of rDNA repeats. Thus, the topographic distribution of rDNA and $\alpha 13,14,21$ repeats in nucleolus is more sensitive to shortage of the hNopp140 than do the nucleolar transcription-related factors.
Fig. 8 RNAi knockdown of hNopp140. HeLa cells were transfected with siRNA duplex specifically targeting the hNopp140 mRNA. Cells were analyzed $72 \mathrm{~h}$ after transfection. Protein levels of hNopp140 in each individual cells were monitored by immunofluorescence staining with the specific mAb. (a and $\mathbf{b}$ ) Double immunofluorescence. UBF and pol I were not affected after down-regulation of hNopp140. (c and d) FISH analysis. Both of the rDNA and $\alpha 13,14,21$ were dispersed away from the nucleolus if hNopp140 was down regulated. (e) Br-UTP incorporation assay. Br-UTP was incorporated in permeabilized cells for $10 \mathrm{~min}$ after the siRNA treatment. The nascent Br-UTP labeled RNA was detected by anti-Br-UTP. The rDNA transcription was arrested only in cells with hNopp140 having been successfully down regulated. Bar, $10 \mu \mathrm{m}$
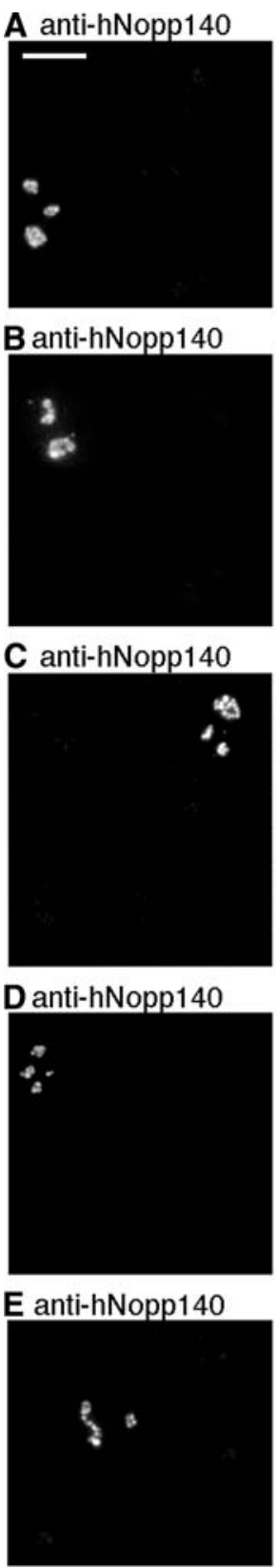
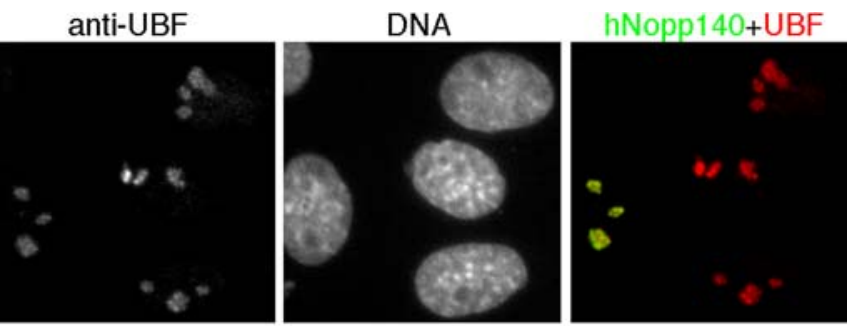

anti-pol I

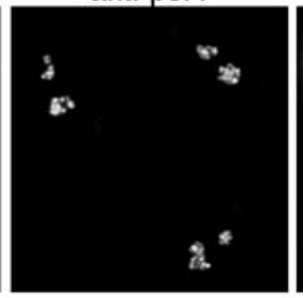

DNA
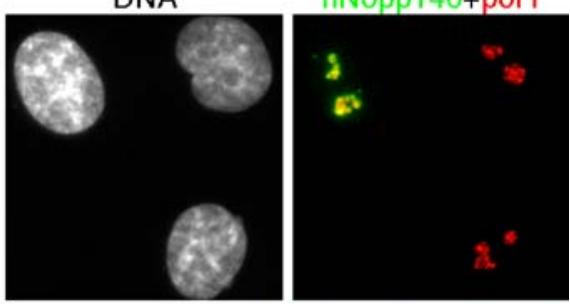

$\alpha 13,14,21$
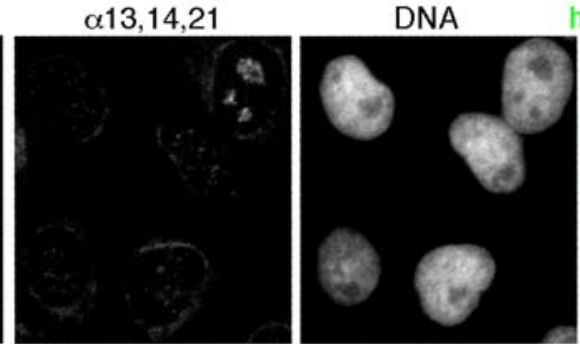

hopp 140+a13,14,21

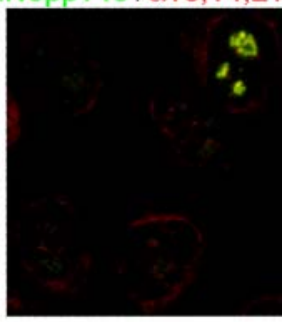

rDNA

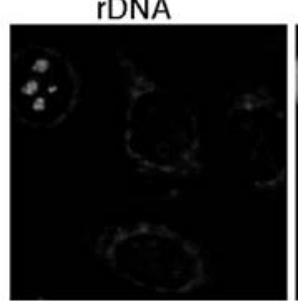

DNA

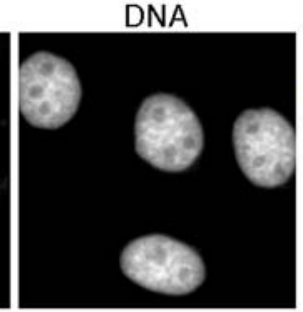

hNopp140+rDNA

anti-Br-UTP

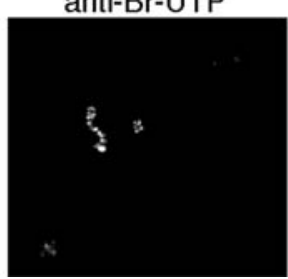

DNA
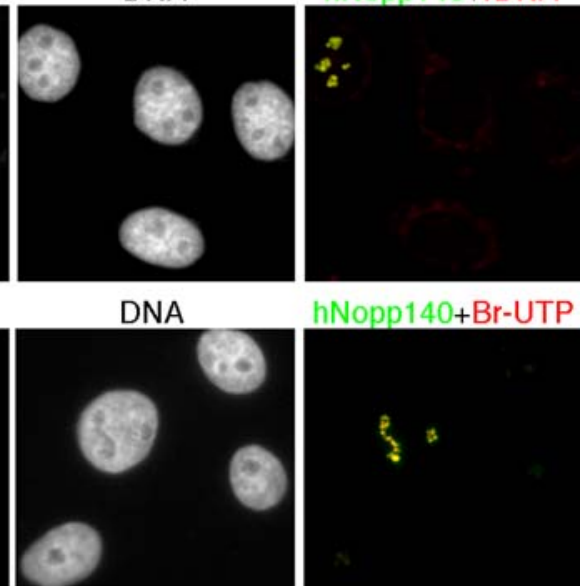

hNopp140+Br-UTP

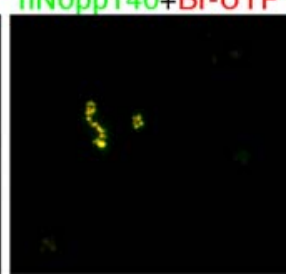




\section{Discussion}

Nucleoli originate from chromosomes at sites containing rDNA repeats. The NOR-associated UBF and pol I serve as docking sites for nucleolar reformation at the exit of mitosis [47]. Moreover, the rDNA chromatin specified by extensive UBF binding can recruit pol I transcription and pre-rRNA processing machineries for coordinating the early events of ribosome biogenesis [48]. However, there must be other components with inherent affinities responsible for spatial organization of rDNA repeats and for nucleolar fusion during nucleologenesis. In this dynamic process, the multiple NORs are drawn to the proximity for facilitating the formation of one or a few large nucleoli [16, $49,50]$. Furthermore, pol I-driven transcription per se is not sufficient for the maintenance of nucleolar morphology [51]. The work described in this paper has revealed that hNopp140 is involved in the nucleolar assembly and maintenance as mediated by tethering of both rDNA and acrocentric $\alpha$-satellite sequences.

Domains for binding to the acrocentric $\alpha$-satellite and rDNA chromatin regions have been defined at the $\mathrm{N}$ - and C-termini of hNopp140, respectively. Without the ten central repeats of hNopp140, the deletion mutants, only when the $\mathrm{N}$ - and $\mathrm{C}$-terminal domains are joined together as represented by DM9, can be concentrated in the nucleolar chromatin-rich places and compete away the endogenous hNopp140. Thus, under physiological condition, the two ends of the hNopp140 molecule are likely folded to a proximal position to create an anchoring domain. Unlike the full-length hNopp140, DM9 does not expand the nucleolar region; and the central ten-repeat, as shown by DM3, homogeneously distributes to the nucleolus in a saturable fashion without affecting the nucleolar appearance. Apparently, both the anchoring domain and the central repeats of hNopp140 are required for hNopp140 to establish the nucleolar round-shaped structure. As the anchoring domains from many hNopp140 molecules attach to chromatin containing the rDNA and acrocentric $\alpha$ satellite arrays, their central repeated regions are able to interact with each other, resulting in forming a highly ordered structure that appears normally as multiple adjacent islets, which can be expanded and fused to become one large sphere if hNopp140 is in large excess. This concept has been supported by the fact that, in addition to the chromatin binding features, hNopp140 has a central repeated domain for homologous self-self interaction. Furthermore, the hNopp140 mutants missing either the $\mathrm{N}$ - or the C-terminal domains have shown the strongly dominant-negative effects to mislocalize the acrocentric $\alpha$-satellite, rDNA repeats, pol I and UBF resulting in blockade of rDNA transcription. Apparently, hNopp140, rDNA repeats and acrocentric $\alpha$-satellite sequences are all involved in forming the nucleolar dotted structure, which is equivalent to the FC and the surrounding DFC [21, 26].

It has been proposed that a nucleolar factory with an FC at its core contains about four transcriptionally active rDNA units [52]. There are $\sim 150$ actively transcribed ribosomal genes per HeLa nucleus [53]. About 10-40 rDNA foci (or nucleolar factories) on the light microscopy level have been mapped in one HeLa nucleus, in which the in situ length of a contorted transcribing rDNA (the $13 \mathrm{~kb}$ coding region) has been calculated to be $\sim 800 \mathrm{~nm}$ on the electron microscopy level [21]. Thus, the rDNA repeats on one chromosome can extend to a considerable distance in nucleus. It is conceivable that these rDNA units even coming from different acrocentric chromosomes are possibly assembled into one round-shaped islet. In each nucleus there are tens of islets, which together may have actually cross-linked all or most of the acrocentric chromosomes. We propose that the assembly of the islets is mediated, at least in part, through hNopp140, due to its unique nature of auto-interaction and recognition of common elements present in the rDNA loci as well as in the centromeric regions of acrocentric chromosomes. Once these chromosomes are cross-linked by the assembled islets during interphase, nucleolar fusion (i.e., multiple NORs to be joined into one nucleolus) would be the consequence.

The evolutionally conserved $\mathrm{N}$ - and C-termini of hNopp140 appear to recognize two distinct DNA sequence elements. One element recognized by the $\mathrm{C}$-terminus of hNopp140 locates within the rDNA cluster region, since the tail-less mutant, DM1, renders the rDNA clusters to be free and to be squeezed out. The transcription-active rDNA repeats associated with nascent rRNA transcripts, seen as the "Christmas trees", are free of regularly spaced nucleosomes [54, 55], and are mobile during transcription in a template-sliding model [52]. Therefore, we interpret our results that these less compact rDNA clusters may be released from the gradually enlarged DM1-containing sphere and eventually condensed into one or two tiny spots that occupy spaces at poles of the sphere. In this context, the DM1 molecules missing only the C-termini can still recognize a second type of elements in nucleolus, likely the acrocentric $\alpha$-satellite arrays, through their $\mathrm{N}$-termini, and expand the nucleolar territory via the homologous interaction of the central repeated domains until the large concave spheres appear. Conversely, the head-less mutant molecules of hNopp140, DM2, unleash the acrocentric $\alpha$ satellite sequences while bringing the rDNA repeats over the DM2-dense region to yield the nucleolar large spheres with asymmetric morphology. In situ run-on transcription assays also support that the DM1 and DM2 with their different partitioning effects can alter locations of the rDNA transcription. Taken together, the $\mathrm{N}-$ or the C-terminally truncated hNopp140 can induce nucleolar 
segregation mediated by detaching the rDNA repeats from the acrocentric $\alpha$-satellite arrays.

The siRNA-mediated blockade of hNopp140 expression reduces but not completely depletes the endogenous hNopp 140, possibly due to its slow turnover rate at the protein level, that is consistent with a report using a different targeting sequence for Nopp140 knockdown [36]. Our results indicate that when the amount of hNopp140 is below a certain threshold, both rDNA repeats and $\alpha 13,14,21$ arrays do not stably stay in the nucleolus. Although it is possible that other components may also be involved in mediating the nucleolar retention of these two DNA sequences, hNopp140 seems relatively more important for rDNA and $\alpha 13,14,21$ to be maintained in nucleolus.

The UBF has been shown to bind to the entire rDNA clusters [56] and to cause chromatin relaxation [57, 58]. Interestingly, we found that greatly overexpressed DM9 induced global chromatin condensation that was accompanied with the dispersion of UBF, hNopp140 and acrocentric $\alpha$-satellite arrays. Under such conditions, the pol I changed its positioning status from a loosely punctate into a densely compact distribution manner, and so did the rDNA. Release of UBF from the rDNA clusters may contribute to condensation of this region. Moreover, the observation of large-scale chromatin condensation across the entire genome during interphase indicates that DM9 can modulate the general chromatin structure. A logical speculation would be that, in addition to the specific recognition of the chromatin regions of the rDNA and the $\alpha 13,14,21$, DM9 might also have a weak binding ability to sites representing a common chromatin configuration. Once the specific nucleolar binding sites have been saturated by DM9, the excessive DM9 accumulated in the nucleoplasm is able to interact with chromatin and alter general features of the chromatin architecture. It is likely that chromatin region of $\alpha 13,14,21$ arrays can escape from nucleolus and distribute into nucleoplasm owing to the pulling effects through DM9, as mediated by recognition of a specific DNA sequence and a nonspecific chromatin configuration. The exact mechanisms remain to be determined.

The Nopp140-chromatin-UBF complexes are for collecting the polymerase I transcription factors and the pre-rRNA processing/modification machineries to engage in activities during the early ribosome biogenesis $[33,34$, 48]. The rRNA intermediate products are then transferred from the Nopp140-containing islets (FC plus DFC) to the surrounding GC compartment for further maturation. Our current findings suggest that Nopp140 is involved in the assembly, fusion, and maintenance of nucleolus. The proposed model should explain principles of the architectural organization within nucleolus.
Acknowledgments We thank Lester I. Binder for Tau-1 antibody. This research was supported by grants from the National Science Council (NSC 89-2320-B010-110) and the National Health Research Institutes (NHRI-EX90-9003BL, NHRI-EX91-9003BL, NHRI-EX929003BL, NHRI-EX93-9003BL, NHRI-EX94-9003BL).

\section{References}

1. Caburet S, Conti C, Schurra C, Lebofsky R, Edelstein SJ, Bensimon A, (2005) Human ribosomal RNA gene arrays display a broad range of palindromic structures. Genome Res 15:10791085

2. Worton RG, Sutherland J, Sylvester JE, Willard HF, Bodrug S, Dube I, Duff C, Kean V, Ray PN, Schmickel RD (1988) Human ribosomal RNA genes: orientation of the tandem array and conservation of the $5^{\prime}$ end. Science 239:64-68

3. Sakai K, Ohta T, Minoshima S, Kudoh J, Wang Y, de Jong PJ, Shimizu N (1995) Human ribosomal RNA gene cluster: identification of the proximal end containing a novel tandem repeat sequence. Genomics 26:521-526

4. Choo KH, Vissel B, Earle E (1989) Evolution of alpha-satellite DNA on human acrocentric chromosomes. Genomics 5:332-344

5. Greig GM, Willard HF (1992) Beta-satellite DNA: characterization and localization of two subfamilies from the distal and proximal short arms of the human acrocentric chromosomes. Genomics 12:573-580

6. Shiels C, Coutelle C, Huxley C (1997) Contiguous arrays of satellites 1,3 , and $\beta$ form a $1.5-\mathrm{Mb}$ domain on chromosome $22 \mathrm{p}$. Genomics 44:35-44

7. Wang S-Y, Cruts M, Del-Favero J, Zhang Y, Tissir F, Potier MC, Patterson D, Nizetic D, Bosch A, Chen H, Bennett L, Estivill X, Kessling A, Antonarakis SE, van Broeckhoven C (1999) A high-resolution physical map of human chromosome 21p using yeast artificial chromosomes. Genome Res 9:1059-1073

8. Scheer U, Thiry M, Goessens G (1993) Structure, function and assembly of the nucleolus. Trends Cell Biol 3:236-241

9. Olson MOJ, Dundr M, Szebeni A (2000) The nucleolus: an old factory with unexpected capabilities. Trends Cell Biol 10: 189-196

10. Hernandez-Verdun D, Roussel P, Gébrane-Younès J (2002) Emerging concepts of nucleolar assembly. J Cell Sci 115:22652270

11. Lam YW, Trinkle-Mulcahy L, Lamond AI (2005) The nucleolus. J Cell Sci 118: 1335-1337

12. Boisvert F-M, van Koningsbruggen S, Navascués J, Lamond AI (2007) The multifunctional nucleolus. Nat Rev Mol Cell Biol 8:574-585

13. Kaplan FS, Murray J, Sylvester JE, Gonzalez IL, O'Connor JP, Doering JL, Muenke M, Emanuel BS, Zasloff MA (1993) The topographic organization of repetitive DNA in the human nucleolus. Genomics 15:123-132

14. Lee W, Kim Y, Lee KY, Kang CS, Lee W, Lee KS, Shim SI, Han $\mathrm{K}$ (1999) AgNOR of human interphase cells in relation to acrocentric chromosomes. Cancer Genet Cytogenet 113:14-18

15. Carvalho C, Pereira HM, Ferreira J, Pina C, Mendonça D, Rosa AC, Carmo-Fonseca M (2001) Chromosomal G-dark bands determine the spatial organization of centromeric heterochromatin in the nucleus. Mol Biol Cell 12:3563-3572

16. Savino TM, Gébrane-Younès J, De Mey J, Sibarita J-B, Hernandez-Verdun D (2001) Nucleolar assembly of the rRNA processing machinery in living cells. J Cell Biol 153:1097-1110

17. Jackson DA, Iborra FJ, Manders EMM, Cook PR (1998) Numbers and organization of RNA polymerases, nascent transcripts, 
and transcription units in HeLa nuclei. Mol Biol Cell 9: 1523-1536

18. Kalmárová M, Smirnov E, Mašata M, Koberna K, Ligasová A, Popov A, Raška I (2007) Positioning of NORs and NOR-bearing chromosomes in relation to nucleoli. J Struct Biol 160:49-56

19. Hernandez-Verdun D (2006) Nucleolus: from structure to dynamics. Histochem Cell Biol 125:127-137

20. Derenzini M, Thiry M, Goessens G (1990) Ultrastructural cytochemistry of the mammalian cell nucleolus. J Histochem Cytochem 38:1237-1256

21. Koberna K, Malínsky J, Pliss A, Mašata M, Vecerová J, Fialová M, Bednár J, Raška I (2002) Ribosomal genes in focus: new transcripts label the dense fibrillar components and form clusters indicative of "Christmas trees" in situ. J Cell Biol 157:743-748

22. Raška I (2003) Oldies but goldies: searching for Christmas trees within the nucleolar architecture. Trends Cell Biol 13:517-525

23. Lazdins IB, Delannoy M, Sollner-Webb B (1997) Analysis of nucleolar transcription and processing domains and pre-rRNA movements by in situ hybridization. Chromosoma 105:481-495

24. Puvion-Dutilleul F, Puvion E, Bachellerie J-P (1997) Early stages of pre-rRNA formation within the nucleolar ultrastructure of mouse cells studied by in situ hybridization with a $5^{\prime}$ ETS leader probe. Chromosoma 105:496-505

25. Puvion-Dutilleul F, Bachellerie J-P Puvion E (1991) Nucleolar organization of HeLa cells as studied by in situ hybridization. Chromosoma 100:395-409

26. Thiry M, Cheutin T, O'Donohue M-F, Kaplan H, Ploton D (2000) Dynamics and three-dimensional localization of ribosomal RNA within the nucleolus. RNA 6:1750-1761

27. Meier UT, Blobel G (1992) Nopp140 shuttles on tracks between nucleolus and cytoplasm. Cell 70:127-138

28. Pai C-Y, Chen H-K, Sheu H-L, Yeh N-H (1995) Cell cycledependent alterations of a highly phosphorylated nucleolar protein p130 are associated with nucleologenesis. J Cell Sci 108:1911-1920

29. Cairns C, McStay B (1995) Identification and cDNA cloning of a Xenopus nucleolar phosphoprotein, $x$ Nopp180, that is the homolog of the rat nucleolar protein Nopp140. J Cell Sci 108:3339-3347

30. Waggener JM, DiMario PJ (2002) Two splice variants of Nopp140 in Drosophila melanogaster. Mol Biol Cell 13:362-381

31. Kelly S, Singleton W, Wickstead B, Ersfeld K, Gull K (2006) Characterization and differential nuclear localization of Nopp140 and a novel Nopp140-like protein in Trypanosomes. Eukaryot Cell 5:876-879

32. Meier UT (1996) Comparison of the rat nucleolar protein Nopp140 with its yeast homolog SRP40. J Biol Chem 271:19376-19384

33. Chen H-K, Pai C-Y, Huang J-Y, Yeh N-H (1999) Human Nopp140, which interacts with RNA polymerase I: implications for rRNA gene transcription and nucleolar structural organization. Mol Cell Biol 19:8536-8546

34. Yang Y, Isaac C, Wang C, Dragon F, Pogacic V, Meier UT (2000) Conserved composition of mammalian Box H/ACA and Box C/D small nucleolar ribonucleoprotein particles and their interaction with the common factor Nopp140. Mol Biol Cell 11:567-577

35. Wang C, Query CC, Meier UT (2002) Immunopurified small nucleolar ribonucleoprotein particles pseudouridylate rRNA independently of their association with phosphorylated Nopp140, Mol. Cell Biol 22:8457-8466

36. Watkins NJ, Lemm I, Ingelfinger D, Schneider C, Hoßbach M, Urlaub H, Lührmann R (2004) Assembly and maturation of the U3 snoRNP in the nucleoplasm in a large dynamic multiprotein complex. Mol Cell 16:789-798

37. Isaac C, Yang Y, Meier UT (1998) Nopp140 functions as a molecular link between the nucleolus and the coiled bodies. J Cell Biol 142:319-329
38. Loomis PA, Howard TH, Castleberry RP, Binder LI (1990) Identification of nuclear $\tau$ isoforms in human neuroblastoma cells. Proc Natl Acad Sci USA 87:8422-8426

39. Lin H-H, Hsu H-L, Yeh N-H (2007) Apoptotic cleavage of NuMA at the C-terminal end is related to nuclear disruption and death amplification. J Biomed Sci 14:681-694

40. Rocchi M, Archidiacono N, Ward DC, Baldini A (1991) A human chromosome 9-specific alphoid DNA repeat spatially resolvable from satellite 3 DNA by fluorescent in situ hybridization. Genomics 9:517-523

41. Soloviev IV, Yurov YB, Vorsanova SG, Malet P (1994) Microwave activation of fluorescence in situ hybridization: a novel method for rapid chromosome detection and analysis. Focus 16:115-116

42. Brown K (2002) Visualizing nuclear proteins together with transcribed and inactive genes in structurally preserved cells. Methods 26:10-18

43. Weinmann AS, Farnham PJ (2002) Identification of unknown target genes of human transcription factors using chromatin immunoprecipitation. Methods 26:37-47

44. Weipoltshammer K, Schöfer C, Almeder M, Philimonenko VV, Frei K, Wachtler F, Hozák P (1999) Intranuclear anchoring of repetitive DNA sequences: centromeres, telomeres, and ribosomal DNA. J Cell Biol 147:1409-1418

45. Ochs RL, Press RI (1992) Centromere autoantigens are associated with the nucleolus. Exp Cell Res 200:339-350

46. Sjöberg MK, Shestakova E, Mansuroglu Z, Maccioni RB, Bonnefoy E (2006) Tau protein binds to pericentromeric DNA: a putative role for nuclear tau in nucleolar organization. J Cell Sci 119:2025-2034

47. Leung AKL, Gerlich D, Miller G, Lyon C, Lam YW, Lleres D, Daigle N, Zomerdijk J, Ellenberg J, Lamond AI (2004) Quantitative kinetic analysis of nucleolar breakdown and reassembly during mitosis in live human cells. J Cell Biol 166:787-800

48. Prieto J-L, McStay B (2007) Recruitment of factors linking transcription and processing of pre-rRNA to NOR chromatin is UBF-dependent and occurs independent of transcription in human cells. Genes Dev 21:2041-2054

49. Carmo-Fonseca M, Mendes-Soares L, Campos I (2000) To be or not to be in the nucleolus. Nat Cell Biol 2:E107-E112

50. Sullivan GJ, Bridger JM, Cuthbert AP, Newbold RF, Bickmore WA, McStay B (2001) Human acrocentric chromosomes with transcriptionally silent nucleolar organizer regions associate with nucleoli. EMBO J 20:2867-2877

51. Raška I, Shaw PJ, Cmarko D (2006) Structure and function of the nucleolus in the spotlight. Curr Opin Cell Biol 18:325-334

52. Cook PR (1999) The organization of replication and transcription. Science 284:1790-1795

53. Jackson DA, Hassan AB, Errington RJ, Cook PR (1993) Visualization of focal sites of transcription within human nuclei. EMBO J 12:1059-1065

54. Trendelenburg MF, Zatsepina OV, Waschek T, Schlegel W, Tröster H, Rudolph D, Schmahl G, Spring H (1996) Multiparameter microscopic analysis of nucleolar structure and ribosomal gene transcription, Histochem. Cell Biol 106:167-192

55. Derenzini M, Pasquinelli G, O'Donohue M, Ploton D, Thiry M (2006) Structural and functional organization of ribosomal genes within the mammalian cell nucleolus. J Histochem Cytochem 54:131-145

56. O'Sullivan AC, Sullivan GJ, McStay B (2002) UBF binding in vivo is not restricted to regulatory sequences within the vertebrate ribosomal DNA repeat. Mol Cell Biol 22:657-668

57. Chen D, Belmont AS, Huang S (2004) Upstream binding factor association induces large-scale chromatin decondensation. Proc Natl Acad Sci USA 101:15106-15111

58. Mais C, Wright JE, Prieto J-L, Raggett SL, McStay B (2005) UBF-binding site arrays form pseudo-NORs and sequester the RNA polymerase I transcription machinery. Genes Dev 19:50-64 